\title{
Dynamic roughness perturbation of a turbulent boundary layer
}

\author{
I. Jacobi $\uparrow$ and B. J. McKeon \\ Graduate Aerospace Laboratories, California Institute of Technology, Pasadena, CA 91125, USA
}

(Received 27 December 2010; revised 30 August 2011; accepted 5 September 2011; first published online 27 October 2011)

The zero-pressure-gradient turbulent boundary layer over a flat plate was perturbed by a temporally oscillating, spatial impulse of roughness, and the downstream response of the flow field was interrogated by hot-wire anemometry and particleimage velocimetry. The key features common to impulsively perturbed boundary layers, as identified in Jacobi \& McKeon (J. Fluid Mech., 2011), were investigated, and the unique contributions of the dynamic perturbation were isolated by contrast with an appropriately matched static impulse of roughness. In addition, the dynamic perturbation was decomposed into separable large-scale and small-scale structural effects, which in turn were associated with the organized wave and roughness impulse aspects of the perturbation. A phase-locked velocity decomposition of the entire downstream flow field revealed strongly coherent modes of fluctuating velocity, with distinct mode shapes for the streamwise and wall-normal velocity components. Following the analysis of McKeon \& Sharma (J. Fluid Mech., vol. 658, 2010, pp. 336-382), the roughness perturbation was treated as a forcing of the Navier-Stokes equation and a linearized analysis employing a modified Orr-Sommerfeld operator was performed. The experimentally ascertained wavespeed of the input disturbance was used to solve for the most amplified singular mode of the Orr-Sommerfeld resolvent. These calculated modes were then compared with the streamwise and wall-normal velocity fluctuations. The discrepancies between the calculated Orr-Sommerfeld resolvent modes and those experimentally observed by phase-locked averaging of the velocity field were postulated to result from the violation of the parallel flow assumption of Orr-Sommerfeld analysis, as well as certain non-equilibrium effects of the roughness. Additionally, some difficulties previously observed using a quasilaminar eigenmode analysis were also observed under the resolvent approach; however, the resolvent analysis was shown to provide reasonably accurate predictions of velocity fluctuations for the forced Orr-Sommerfeld problem over a portion of the boundary layer, with potential applications to designing efficient flow control strategies. The combined experimental and analytical effort provides a new opportunity to examine the non-equilibrium and forcing effects in a dynamically perturbed flow.

Key words: turbulent boundary layers 


\section{Background}

Non-equilibrium boundary layers are of significant interest for a variety of practical flows, as reviewed by Smits \& Wood (1985) and more broadly by Morrison (2010), but most previous work has considered non-equilibria in the form of a static impulseresponse or step. Smits, Young \& Bradshaw (1979) considered abrupt geometric changes to a flat plate, in the form of short convex or concave regions, while Antonia \& Luxton (1972) and Andreopoulos \& Wood (1982) considered abrupt changes in surface roughness. Equilibrium is understood in the local energetic sense, as used by Townsend (1961). In the roughness studies, internal layers were identified which mark the extent to which the mean flow downstream of a transition in roughness adapts to the new boundary condition. At a smooth to rough transition $(S \rightarrow R)$, a fast-growing internal layer, $\delta_{1}$, was observed which quickly resulted in re-equilibration of the flow, whereas at a rough to smooth transition $(R \rightarrow S)$, a more slowly growing internal layer, $\delta_{2}$, appeared to persist more than $16 \delta_{0}$ downstream of the impulse, where $\delta_{0}$ represents $99 \%$ of the mean incoming boundary layer thickness.

More recently, Jacobi \& McKeon (2011) reconsidered the case of a short impulse of static roughness on an otherwise smooth flat plate, and were able to place a number of the key observations of previous experimenters into a simple and physically motivated framework. By building on the idea of the 'stress bore' introduced by Smits et al. (1979) in the context of abrupt bends in otherwise flat plates, it was shown that the boundaries of the internal layers generated by a static impulse of roughness roughly demarcate a region of the downstream flow field which behaves as a bore of shear stress. The alteration of the flow in this region was shown to manifest itself in the streamwise velocity statistical moments (consistent with Andreopoulos \& Wood 1982), which in turn were shown to scale purely on the local shear stress conditions, independent of the wall boundary. The local nature of the bore scaling then explains the unusually long persistence of the perturbation, far downstream of the impulse, and also its eventual weakening and diffusion. In addition, it was shown that the static impulse of roughness had a significant effect on the spectral signature of the near-wall cycle in the immediate vicinity of the perturbation, in agreement with the flow visualizations of Pearson, Elavarasan \& Antonia (1997); this effect was quantified by examining the redistribution of streamwise, turbulent spectral energy density in the wall-normal direction. Also, the impulse produced a persistent impact on the vortical structure of the downstream flow. The essential idea of a short impulse of roughness can then be thought of as the injection of a spatial scale, associated with the roughness, into the flow. The flow then relaxes, in a complex way governed by at least two independent time scales, one for the evolution of the stress bore and one for the near-wall cycle interruption and recovery near the perturbation.

However, if instead of a purely static impulse, the impulse is dynamic, with an associated time scale, then in addition to the spatial scale of the roughness, the time scale can also be injected into the flow field. In this way, a more detailed understanding of the mechanics of the relaxation can be obtained, since the relaxation processes observed in the static impulse can be viewed in the context of a particular input time scale. The development of the stress bore and the redistribution of turbulent spectral energy density downstream of the dynamic perturbation, in contrast to the statically perturbed case, can be used to better understand the important time scales in non-equilibrium flow conditions. Moreover, the extent to which the dynamic wave associated with the periodic oscillation of the roughness strip, and the spatially impulsive roughness effects themselves, can be treated separately is also considered. 
Beyond the experimental comparisons of the dynamic roughness perturbation to its static counterpart, the dynamic perturbation is also amenable to modelling, allowing predictions of the velocity fluctuations generated by the perturbation. Early modelling of the experimental, dynamic perturbation of a turbulent channel flow was undertaken by Hussain \& Reynolds (1970). They utilized a thin oscillating ribbon near the wall of a channel to disturb a turbulent base flow at a fixed frequency and amplitude and observed the evolving properties of the perturbation downstream. Reynolds \& Hussain (1972) subsequently considered various turbulence modifications to the Orr-Sommerfeld equation which governs small perturbations to the Navier-Stokes equations, in order to model their experimental observations. In particular they considered what they called a 'quasi-laminar' closure scheme, wherein the Reynolds stresses were neglected and the turbulence appeared in the model only via the turbulent velocity profile, as well as a number of eddy viscosity approaches, and using these approaches, they estimated the wavenumbers of the perturbations expected in the flow. They concluded that inclusion of the Reynolds stress terms was essential for accurate predictions and that the quasi-laminar approach failed in two respects: (a) it was unable to accurately predict the wavespeeds (eigenvalues) experimentally measured, and $(b)$ the mode shapes of velocity fluctuations (eigenfunctions) predicted were disproportionately more peaked than those observed in the experiments.

However, the modelling efforts of Reynolds \& Hussain (1972) deserve reconsideration in light of the recent work of McKeon \& Sharma (2010) which applied a 'resolvent analysis' to the Orr-Sommerfeld equation associated with turbulent pipe flow. The resolvent analysis shares the same starting point as the traditional eigenvalue analysis of the Orr-Sommerfeld equation, by applying a Reynolds decomposition to the Navier-Stokes equation, but instead of linearizing the result, the nonlinear terms are retained and grouped on the right-hand side as an 'internal forcing', $f$. In this way, the model equation is restructured to appear like the standard Orr-Sommerfeld linear eigenvalue problem on the left-hand side, but with 'internal forcing' on the right-hand side representing the natural nonlinearities inherent in the flow. By inverting the linear operator, the problem was shown to be equivalent to the resolvent (or propagator) of the Orr-Sommerfeld operator acting on the 'internal forcing', $f$. McKeon \& Sharma (2010) proposed that the most-amplified singular mode of the resolvent, formulated for particular combinations of streamwise and spanwise wavenumbers and frequencies, could be used to represent key features of the overall flow field, in much the way that proper orthogonal decomposition (POD) methods employ singular modes to identify reduced-order representations of complicated flow fields (Hellström \& Smits 2011). The dominant modes were identified as the turbulent analogues of the well-studied 'critical' and 'wall' (upper and lower branch) neutral disturbances in linear stability analysis. Importantly, the standard closure problem was circumvented, to the extent that one or more singular modes superposed can represent the flow field, and only the mean turbulent velocity profile is needed for construction of the Orr-Sommerfeld resolvent.

In the current investigation, when forcing is introduced to the flow field, the righthand side of the governing equation includes not only the natural nonlinearities of the unforced problem, labelled 'internal forcing', but also the 'external forcing' injected into the flow. The resolvent approach applied to this forced problem differs from that of the quasi-laminar approach of Reynolds \& Hussain (1972) in a number of significant ways.

The most obvious difference between the approaches is the type of modal decomposition assumed. Because the Orr-Sommerfeld operator is not normal - a 
property which Trefethen \& Embree (2005) note was not widely appreciated for most of the history of its study - its corresponding eigenfunctions are not orthogonal and its eigenvalues are highly sensitive to small perturbations. The non-orthogonality of the eigenfunctions make them particularly ill-suited for representation of high-Reynoldsnumber flows, as noted by Schmid \& Henningson (2001), a problem made worse in boundary layer flows where the eigenfunctions do not even provably form a complete set, noted in Grosch \& Salwen (1978). On the other hand, the resolvent analysis avoids the entire difficulty associated with eigenvalues, by employing a Schmidt decomposition, which produces an orthogonal set of singular basis functions even for non-normal operators, and the resolvent operator itself, by construction, naturally contains information about the wavespeed of the 'external forcing'.

The resolvent analysis also implicitly treats a superposition of all of the forcing, via the mean velocity profile, and as stated above, requires no explicit closure treatment. The downside of this, however, is that the relative strengths of the 'external forcing' and 'internal forcing' may be important, in terms of the number of modes necessary to adequately describe the flow. In other words, if the perturbation is relatively weak compared to the 'internal forcing' of the natural nonlinearities in the base flow, the perturbed dynamics may not be captured with just a single mode of the resolvent. Of course this problem of the relative strength of the perturbation also afflicts the quasi-laminar approach, and indeed Reynolds \& Hussain (1972) considered a superposition of different eigenmodes to adequately capture the dynamics. The method of roughness perturbation employed in the current approach naturally generates a stronger perturbation to the base flow than was considered in the experiment of Hussain \& Reynolds (1970), which was, by comparison, closer to the very low perturbation level used in transition studies of laminar flows, like that of Schubauer \& Skramstad (1947). This difference in perturbation strength indicates that the contribution of the 'external forcing' to the overall forcing of the problem should be significant in the current experiments (and not overwhelmed by natural nonlinearities in the base turbulent flow), which would allow for the possibility that a small number of singular modes could adequately capture the velocity fluctuations of the forced system. Moreover, the relatively stronger perturbation also provides insight into more realistic forcing generated by various mechanical flow control schemes.

However, ultimately, the primary reason for the disqualification of the quasi-laminar scheme was its inaccurate prediction of eigenmode shapes compared to measurements of velocity fluctuations. Although the resolvent approach entirely avoids the sensitive choice of eigenvalues and modes, certain deficiencies in the mode predictions by the resolvent persist and will be explored and compared with the other approaches.

The present study has two primary objectives: (a) to connect the key features of the statically perturbed boundary layer - the internal layers, stress bore, nearwall energetic changes, and integral scale-size effects - to a dynamic perturbation of similar spatial type; and $(b)$ to utilize that dynamic perturbation to examine the effect of exciting a specific temporal fluctuation on the flow field, by employing a resolvent analysis. Thus this manuscript both presents the experimental measurements and attempts a predictive analysis, in support of the experiments, in order to provide a comprehensive picture of the perturbed flow. Considering the dynamic perturbation in this broader framework offers an experimental basis for a new approach to externally forced boundary layers, with potentially significant applications to flow control.

In $\S 2$, the experimental technique by which the dynamic perturbation was generated and the flow measured is described. Section 3 provides the statistical and spectral view of the perturbed flow, with emphasis on how the dynamic perturbation contrasts with 
the static perturbation considered previously, and the extent to which the roughness and wave-like features of the perturbation can be separated. The wave-like features are studied more carefully in $\S 4$ by employing a phase-locked decomposition of the velocity signal to identify the shapes of the velocity fluctuations in the flow field. Finally, $\S 5$ employs the resolvent method of predicting the fluctuating velocity modes anticipated under the periodic perturbation, and provides additional details on the singular modes associated with the turbulent Orr-Sommerfeld problem.

\section{Experimental method}

The turbulent boundary layer experiments were performed in the $2 \mathrm{ft} \times 2 \mathrm{ft}$ wind tunnel at Caltech, previously described in Jacobi \& McKeon (2011), where a zero-pressure-gradient was maintained by an adjustable ceiling which limited the spatial variation in pressure coefficient $\Delta C_{p} \ll 0.01$ over the range of streamwise measurement locations; thus irrotational effects are assumed negligible. The boundary layer was tripped at the leading edge, far upstream of the position of the dynamic perturbation. The perturbation was implemented through an acrylic insert fitted into the smooth flat plate which allowed a short patch of roughness elements - twodimensional for simplicity - to be articulated through the surface of the plate, while not allowing any cross-flow of air between the surface and underside of the flat plate. The short patch of roughness was identical to that used in the previous work on static perturbations, in order to allow comparison between the two disturbances. Four spanwise bars of two-dimensional, $k$-type roughness, $1.57 \mathrm{~mm}$ thick and separated by $6.35 \mathrm{~mm}$ were affixed to a single array to allow them to move in unison. The patch was situated on the flat plate where the undisturbed incoming flow reached $R e_{\theta}=2770$, and the streamwise extent of the patch was approximately 1.5 times the incoming boundary layer thickness, providing a spatially impulsive disturbance.

Beneath the test section, an armature connected the roughness strip via a piston to a crank-shaft assembly and DC motor (Dayton 4z142, 1/27 h.p. 1800 r.p.m.) which provided a reciprocating motion of the roughness elements with amplitude fixed by the offset of the connecting rod to the centre of the shaft. This offset was fixed nominally at $1 \mathrm{~mm}$, anticipating some small amount of slippage due to wear, such that the actual displacement of the roughness elements (from TDC to BDC) was approximately $k=1.64 \mathrm{~mm}$. The root-mean-square (r.m.s.) height for a periodic motion with this maximum amplitude was therefore $k_{r m s}=1.16 \mathrm{~mm}$, which approximately matched the previously reported case of static roughness elements, which had $k=1.0 \mathrm{~mm}$. In the current experiment, the match in r.m.s. height provided a common degree of timeaveraged blockage between the static and dynamic cases (with an eye towards phaselocked averaging); alternatively, the maximum amplitude could have been matched to achieve a common instantaneous 'impulsive' strength. A magnetic linear encoder (Renishaw LM10) with $1 \mu \mathrm{m}$ spatial resolution was affixed to the roughness armature, which in turn was connected to a Labview-operated quadrature encoder with a $250 \mathrm{MHz}$ internal counter, to resolve the motion of the armature. The encoder signal was then sampled simultaneously with the anemometer signals at $60 \mathrm{kHz}$ in order to allow phase-locked sampling of the anemometer signals by using the reference encoder signal, as described in $\S 4.1$.

A schematic of the experimental setup, along with the relative positions of the different measurement locations, is provided in figure 1. An average period of the peak height of the roughness elements is shown in figure 2, where a height of 0 represents the elements being flush with the remainder of the flat plate, and a positive 

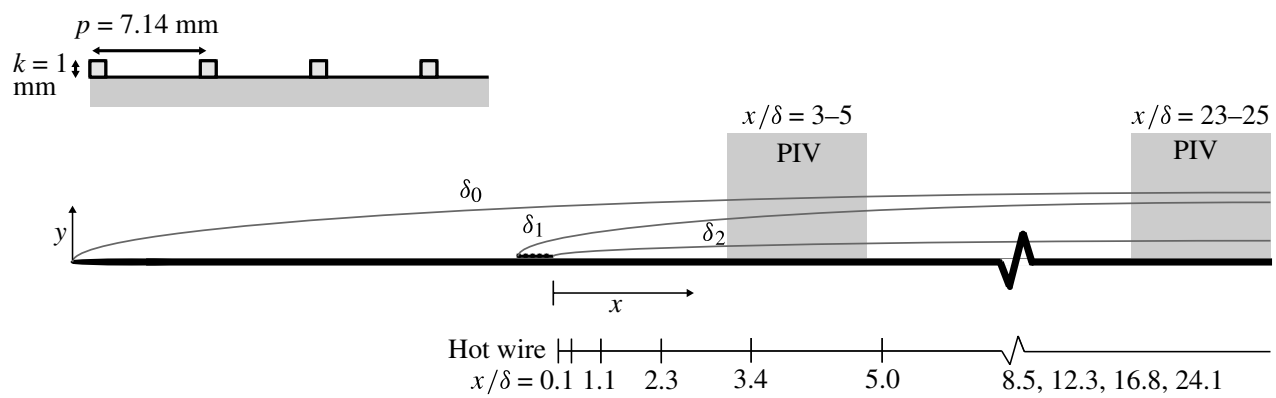

FIGURE 1. A schematic of the arrangement of the flat plate, the roughness strip, and the diagnostic locations; not to scale. The internal layers are also marked in order to provide an idea of the relative size and development rates.

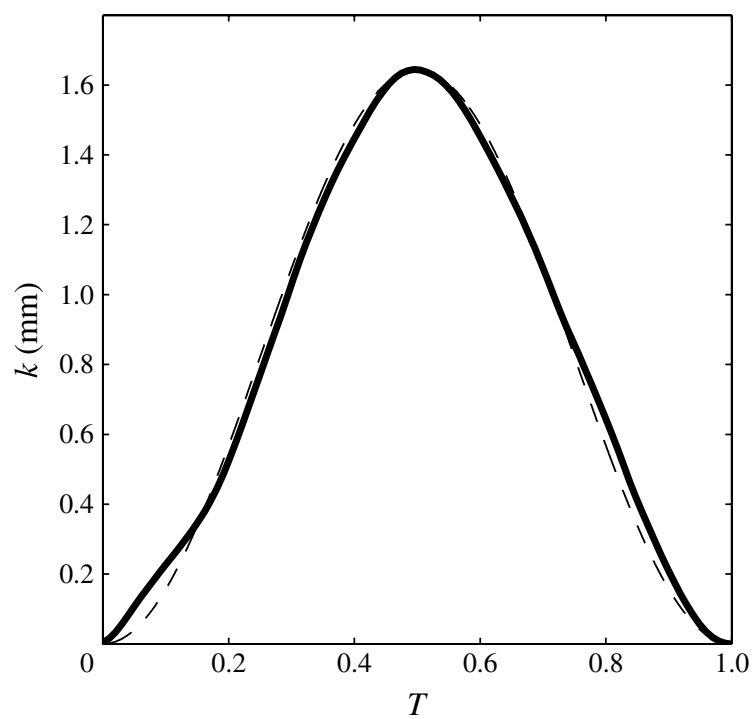

FIGURE 2. An average period, $T$, of the encoder signal, measured by ensemble averaging the mean displacements from each phase of a phase-locked decomposition; the precise method is discussed in $§ 4.1$. - , encoder phase-locked signal; --, undistorted sinusoid.

displacement is the height above the plate. The oscillation was not perfectly sinusoidal due to slippage and frictional non-uniformities in the slots through which the armature reciprocated. The flow field downstream of the roughness impulse was measured by hot-wire anemometry and particle-image velocimetry (PIV), details of which, including validation of the unperturbed flow, have been previously reported by Jacobi \& McKeon (2011).

The measured velocity signal $u(y, t)$, in the following analysis, can be expressed by the standard Reynolds decomposition as

$$
u(y, t)=U(y)+u^{\prime}(y, t),
$$

where the mean profile is $U(y)$ and the turbulent fluctuation about the mean is $u^{\prime}(y, t)$, with free-stream velocity $U_{\infty}$. However, the fluctuation, in principle, can be further decomposed into a periodic component $\tilde{u}(y, t)$ contributed by the periodic input 


$\begin{array}{lccccccc}\text { Wall } & \begin{array}{c}x \\ (\mathrm{~mm})\end{array} & \begin{array}{c}U_{\infty} \\ \left(\mathrm{m} \mathrm{s}^{-1}\right)\end{array} & \begin{array}{c}\delta \\ (\mathrm{mm})\end{array} & \begin{array}{c}\theta \\ (\mathrm{mm})\end{array} & \begin{array}{c}\delta^{*} \\ (\mathrm{~mm})\end{array} & R e_{\theta}=U \theta / v & R e_{\tau}=u_{\tau} \delta_{99} / v \\ \text { Unperturbed } & 5 & 20.16 & 17.0 & 2.1 & 2.9 & 2770(2560) & 910(970) \\ & (7.4) & (20.60) & (17.6) & (1.9) & (2.9) & & \\ & 58 & 20.09 & 24.1 & 3.1 & 4.3 & 4070(3870) & 1200(1320) \\ \text { Static } & (62.4) & (20.42) & (25.8) & (2.9) & (4.3) & & \\ & 2.5 & 20.20 & 17.2 & 2.1 & 3.6 & 2770(2970) & \\ & (7.4) & (20.65) & (18.4) & (2.2) & (3.5) & & \\ & 58 & 20.07 & 24.4 & 3.3 & 4.6 & 4330(4150) & \\ \text { Dynamic } & (62.4) & (20.50) & (26.7) & (3.1) & (4.6) & & \\ & 2.5 & 20.13 & 17.0 & 2.1 & 3.3 & 2770(2990) & \\ & (7.4) & (20.78) & (18.3) & (2.2) & (3.3) & & \\ & 58 & 20.11 & 24.3 & 3.2 & 4.5 & 4330(4160) & \end{array}$

TABLE 1. Mean flow properties at streamwise extrema of sampling area for hot-wire (and PIV). $\theta$ is the momentum thickness; $\delta^{*}$ is the displacement thickness; and $v$ is the kinematic viscosity.

perturbation, and then a turbulent fluctuation about the periodic component, $u_{t}^{\prime}(y, t)$, such that the overall decomposition is

$$
u(y, t)=U(y)+\tilde{u}(y, t)+u_{t}^{\prime}(y, t) .
$$

The details of this phase-locked composition are provided in $\S 4.1$, and for now, the overall fluctuation $u^{\prime}(y, t)$ will be treated. $\sqrt{\overline{u^{\prime 2}}}$ is the root-mean-square value of $u^{\prime}(y, t) ; \sqrt[3]{\overline{u^{\prime 3}(y)}}$ is the cube-root of the third moment of $u^{\prime}(y, t)$. The streamwise position $x$ is measured from the trailing edge of the roughness patch.

\section{General features of the dynamic perturbation}

In this section, the mean flow properties for the unperturbed smooth flow and the flow perturbed by the dynamic impulse are presented, with comparison to a few key results from the static perturbation study. The behaviour of the dynamic impulse is also compared to previous impulsively perturbed flows and the internal layers resulting from the perturbation are identified and interpreted. The effect of the perturbation on the turbulence statistics, the spectral energy density distribution, and the spatial distribution of integral length scales in the downstream flow are all presented and interpreted in light of the time-varying nature of the perturbation. Finally, spectral methods are presented which motivate the division of the effect of the dynamic perturbation into two separate regimes: an impulsive roughness perturbation and an organized wave.

\subsection{Mean flow properties}

The essential flow properties for both the hot-wire and PIV experiments are summarized in table 1, and some key features of the flow field are described below. The dynamically perturbed case refers to a perturbation by the roughness patch when the patch was actuated by the motor; the statically perturbed case refers to the previous study by Jacobi \& McKeon (2011) with the identical roughness elements 
fixed in position at a roughly equivalent amplitude. The streamwise growth rate of the boundary layer thickness was approximately the same for both the static and dynamically perturbed flows, and both perturbed boundary layers grew more quickly than the unperturbed boundary layer. $\delta$ refers to the value of $\delta_{99}$ at a given streamwise position under each flow regime; $\delta_{0}$ is the incoming boundary layer thickness.

The friction velocity $u_{\tau}=\sqrt{\tau_{w} / \rho}$ was estimated by the Clauser method and also independently verified by the momentum integral approach and by inference from the velocity gradient very near the wall. As discussed in the previous work on the static perturbation, neither the Clauser method nor the momentum integral approach strictly applies, due to the non-equilibrium conditions downstream of the perturbation. Calculating the velocity gradient by using the first mean velocity measurement nearest the wall, and a no-slip condition, is also problematic, due to both the uncertainty in the wall position and the location of the first point at the outer edge of the linear regime. Despite these caveats, using this linear-fit technique, the dynamically perturbed flow shows a drop in skin friction, $C_{f}$, immediately downstream of the roughness strip (similar to the static impulse), and then an oscillating recovery (in contrast to the nonoscillatory recovery previously reported for the statically perturbed case). The average spatial period of this oscillation is approximately $6 \delta$, compared to the wavelength of the dynamic perturbation of about $20 \delta$ measured below, suggesting tentatively that the relaxation is decoupled from, or only weakly dependent on, the dynamic impulse and is thus a function of the smaller wavelength structures associated with the roughness. The general trend in the recovery of $C_{f}$, including the overshoot and oscillation, is consistent with previous work on a static roughness impulse by Andreopoulos \& Wood (1982) and Pearson et al. (1997). However, since all of the $C_{f}$ measurement techniques suffer from significant sources of uncertainty, scaling throughout the remaining results is accomplished in terms of outer variables or, when noted, in terms of the inner scales corresponding to the unperturbed case only. An independent measure of $\tau_{w}$ would enable more robust analysis; in particular, the measurement of skin friction in a non-equilibrium boundary layer being forced dynamically in time poses additional measurement challenges and is a topic of current investigation.

The strength of the dynamic perturbation can be quantified in terms of its effect on the roughness function $\Delta U / u_{\tau}$ from the traditional law of the wall, as described in Antonia \& Luxton (1971), by defining a logarithmic ratio, $M$, of the roughness heights at each surface condition determined via the roughness function. Jacobi \& McKeon (2011) discussed a number of challenges to employing this metric for non-equilibrium perturbations, but despite the caveats outlined there, the technique shows the expected result that the strength of the dynamic impulse is weaker than the corresponding impulse in the static case: the $M_{S \rightarrow R}$ transition was approximately -1.0 (versus -1.7 in the statically perturbed flow) and the $M_{R \rightarrow S}$ transition was approximately 1.0 (versus 1.6). Indeed, the ratio of the static to dynamic impulse strengths is $\approx \sqrt{2}$ as would be expected considering that the the impulse strength would scale on the instantaneous maximal amplitude, whereas the time-averaged blockage was selected to be held constant.

The mean velocity profiles were compared between the dynamically perturbed and unperturbed flows. Immediately downstream of the perturbation, there is a significant velocity deficit, particularly for $y / \delta<0.3-0.4$, which corresponds to approximately 6 times the roughness height. This deficit persists until approximately $15 \delta$ downstream of the trailing edge of the perturbation. There appears to be a persistent, albeit small, velocity deficit even further downstream and across the velocity profile, consistent with the results of Andreopoulos \& Wood (1982). By plotting the discrepancy in the mean 

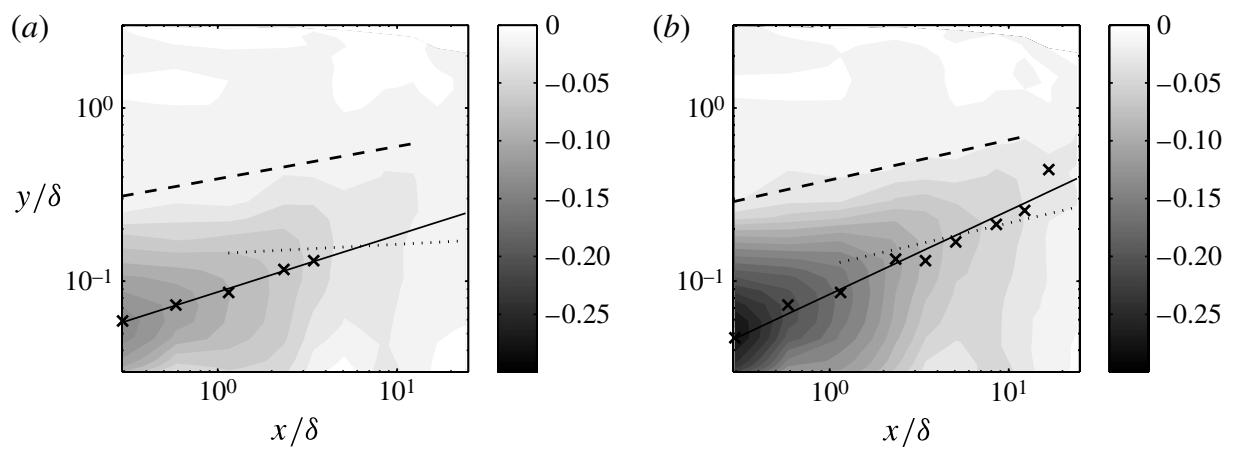

FIGURE 3. (a) The discrepancy in the mean velocity profiles between the dynamically perturbed and unperturbed cases is shown in grey contours; the peaks for each streamwise location at which the velocity discrepancy is greatest are marked $\times$ and a curve is fitted by least squares $\left(-, y / \delta=0.09(x / \delta)^{0.33}\right)$. The approximate intercept for the fit is just below the r.m.s. height of the roughness elements: $y \approx 0.06 \delta=0.88 k_{r m s}$. Also, internal layer best fits, calculated in the text, are included $\left(--, \delta_{1} / \delta=0.39(x / \delta)^{0.19} ; \cdots, \delta_{2} / \delta=0.14(x / \delta)^{0.05}\right)$ for reference. (b) For comparison the statically perturbed case is shown with the least-squares fit for the peak discrepancy given by $y / \delta=0.08(x / \delta)^{0.48}$ with approximate intercept at $y \approx 0.05 \delta=0.86 k$.

velocity profiles between the perturbed and unperturbed cases

$$
\frac{\Delta u}{U}(x, y)=\frac{u}{U}(x, y)_{\text {perturbed }}-\frac{u}{U}(x, y)_{\text {smooth }},
$$

as a contour map in wall-normal and streamwise directions following the procedure from Jacobi \& McKeon (2011), the recovery behaviour of the mean velocity profile can be seen quite clearly in figure 3. Least-squares curve fits for the wall-normal location of the peak velocity deficit at each streamwise measurement location are provided for comparison. The discrepancy map for the static perturbation is also provided for comparison.

The velocity deficit is smaller in both wall-normal and streamwise extent than that of the statically perturbed case, as expected, and thus the recovery appears to occur more quickly, although still on the order of $10 \delta$.

\subsection{Turbulence statistics}

Turbulence statistics were calculated in the streamwise direction from the hot-wire signals. Plotting successive profiles of the streamwise turbulence intensity $\sqrt{\overline{u^{\prime 2}(y)}}$ shows a large 'hump' in the profile, similar to that seen in the statically perturbed case but broader in wall-normal extent and higher in amplitude. This hump had previously been interpreted as a key manifestation of the stress bore generated in the flow by the perturbation. For a dynamic perturbation, this bore is expected to span a broader range of wall-normal locations, since the roughness operates at a range of locations from $y=0 \rightarrow k$, and indeed the hump is seen to extend from near the wall out to the location of the edge of where the hump in the statically perturbed case resides, with its peak centred at $y=0.08 \delta=1.3 \mathrm{k}$ immediately downstream of the roughness. 

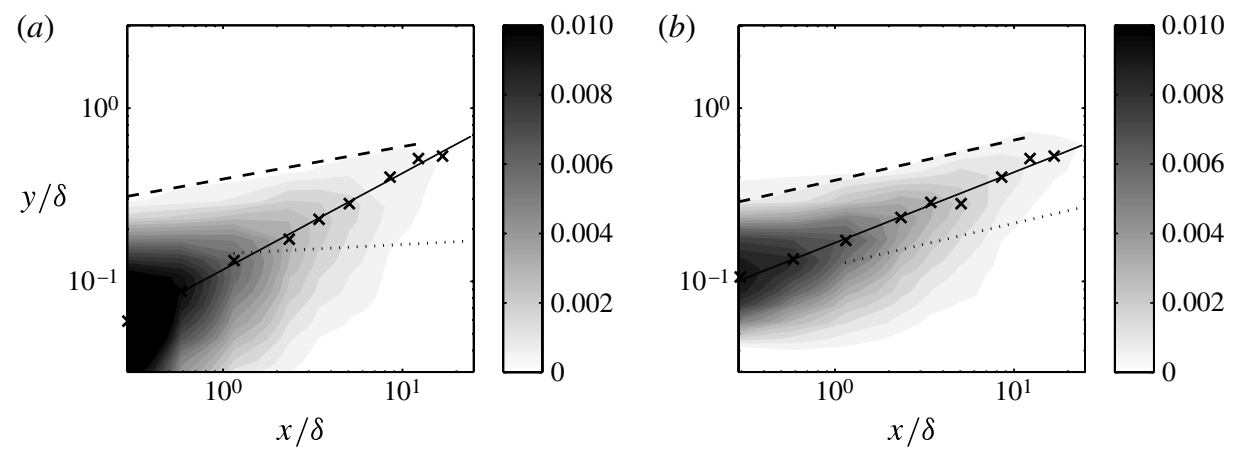

FIGURE 4. (a) The discrepancy in the streamwise turbulence intensity profiles between the perturbed and unperturbed cases is shown in grey contours; the peaks for each streamwise location at which the discrepancy is greatest are marked $x$ and a curve is fitted by least squares $\left(-, y / \delta=0.12(x / \delta)^{0.54}\right)$. The approximate intercept for the fit is the same as for the mean velocity discrepancy: $y \approx 0.06 \delta=0.88 k_{r m s}$. And again, the internal layer boundary best fits, calculated in the text, are included for reference. (b) For comparison, the statically perturbed case is shown, with the least-squares fit for the peak discrepancy given by $y / \delta=0.17(x / \delta)^{0.41}$ with approximate intercept $0.10 \delta=1.67 \mathrm{k}$.

As with the velocity discrepancy contours, the discrepancy in the streamwise turbulence intensity profiles between the perturbed and unperturbed cases

$$
\frac{\Delta \sqrt{\overline{u^{\prime 2}}}}{U}(x, y)=\frac{\sqrt{\overline{{u^{\prime 2}}^{2}}}}{U}(x, y)_{\text {perturbed }}-\frac{\sqrt{\overline{{u^{\prime 2}}^{2}}}}{U}(x, y)_{\text {smooth }},
$$

can be viewed as a contour map in wall-normal and streamwise directions in order to visualize the recovery behaviour of the flow field (figure 4). In this case, the magnitude and streamwise extent of the discrepancy appears comparable between the dynamically perturbed flow and the static perturbation, except in the immediate vicinity of the perturbation itself.

As reported for the static impulse, the hump in the turbulence intensity plots, which varies with streamwise position downstream of the dynamic impulse, is a manifestation of the underlying stress bore, and thus can be scaled by a velocity scale based on the mean velocity gradient, $u_{s}$

$$
u_{s}=\sqrt{\left(U_{\infty} \delta \frac{\partial U}{\partial y}\right)},
$$

which represents the continued influence of the near-wall perturbation even farther from the wall and downstream, governed by local effects.

Under this scaling (figure 5) the hump collapses for streamwise locations $x>2 \delta$, consistent with the idea that the inter-layer region under dynamic perturbation also behaves as a stress bore reflecting the boundary condition enforced for that region. However, for $x \lesssim 2 \delta$, the scaling does not appear to collapse the profiles in the region $y<0.06 \delta<0.88 k_{r m s}$ - in precisely the region nearest the wall that also shows the deviation in the $\sqrt{\overline{u^{\prime 2}(y)}}$ contours between the static and dynamic cases - which indicates that this failure of the $u_{s}$-scaling might reflect a fundamental difference between the two types of perturbation. By plotting the contours of the ratio between $u_{s}$ under the two regimes, in figure 6, two distinct regions become clear. Between 

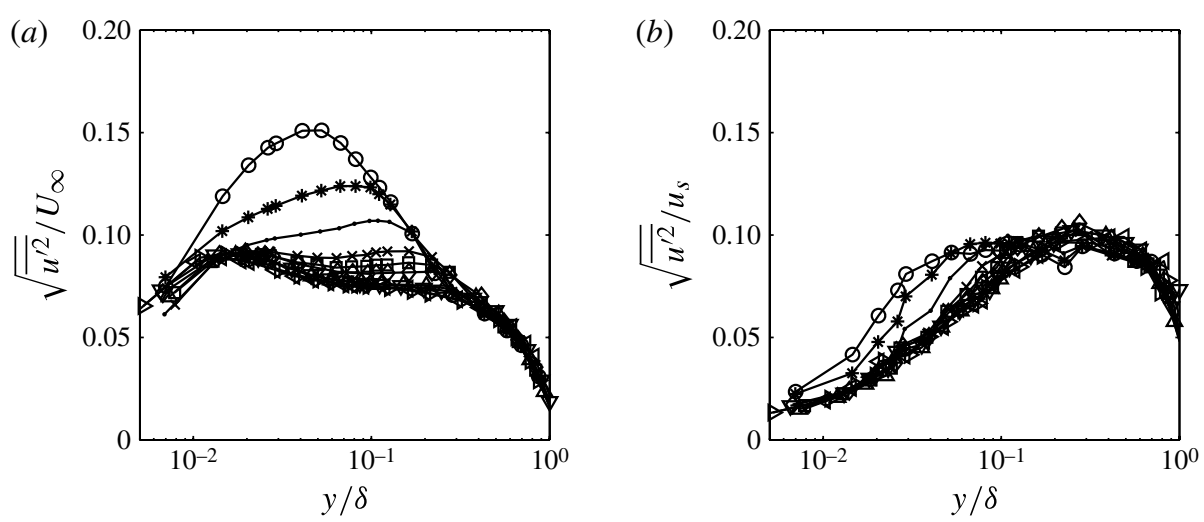

FIGURE 5. The turbulence intensity for the perturbed flow under (a) standard outer scaling; and $(b)$ under a velocity scaling based on the local shear stress, $u_{s}$. Streamwise profiles: $\bigcirc, x / \delta=0.3 ; *, 0.6 ; \cdot, 1.1 ; \times, 2.3 ; \square, 3.4 ; \diamond, 5.0 ; \triangle, 8.4 ; \nabla, 12.1 ; \triangleright, 16.6 ; \triangleleft, 23.8$.

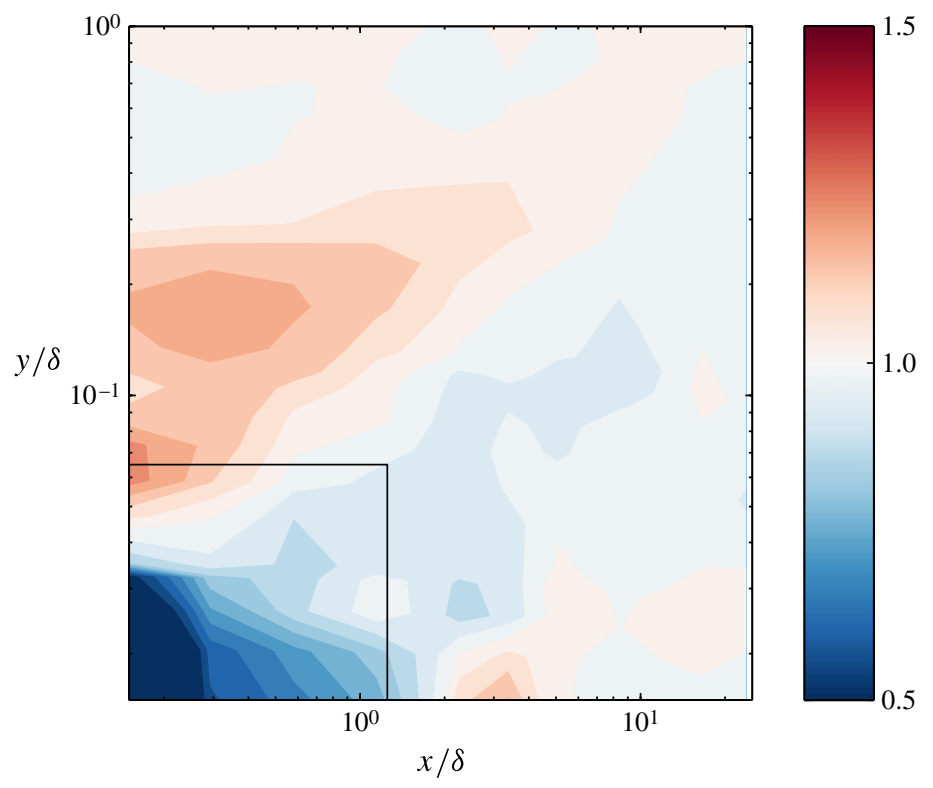

FIGURE 6 . The ratio of $u_{s}$ for the static impulse over $u_{s}$ for the dynamic impulse. The rectangle represents the area in figure 5 where the $\sqrt{\overline{{u^{\prime 2}}^{2}(y)}}$ profile fails to collapse.

the mean edges of the two internal layers, the value of $u_{s}$ is $\sim 30 \%$ larger in the static than dynamic case, consistent with the expected result from a roughness effect corrected for the r.m.s. roughness height. In the region in the immediate vicinity of the roughness elements, however, the ratio is reversed. The velocity gradient of the dynamically perturbed case dominates here, perhaps due to the oscillating roughness elements - which generates a much higher shear locally about the trailing edge of the last element. In this region, it is the dynamic (or long-wavelength) feature of the oscillation which dominates, as opposed to the more permanent feature of the spatial impulse seen farther from the wall. Thus we expect the reverse trend, that the mean 


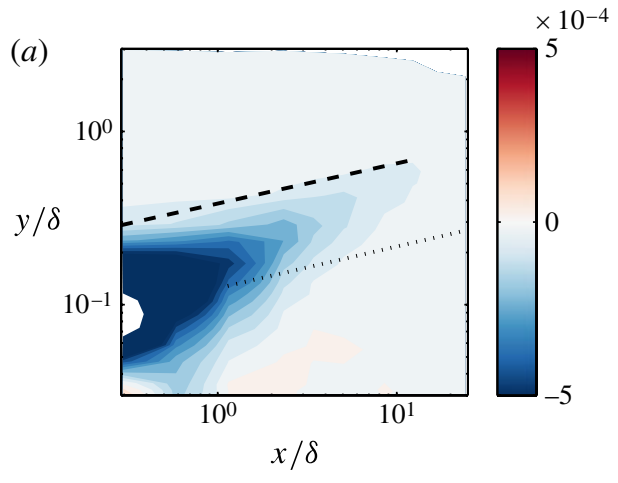

(b)

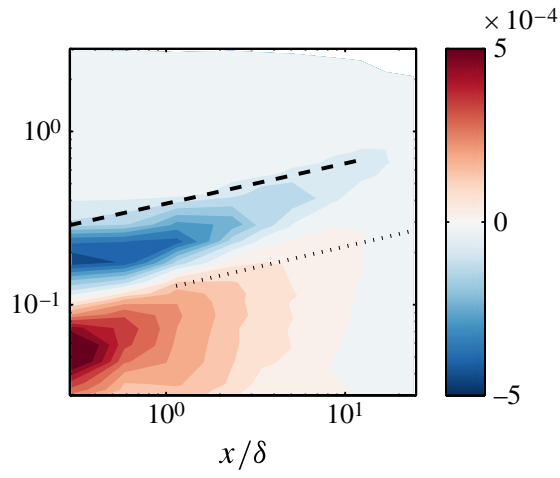

FIGURE 7. The discrepancy map for the streamwise velocity triple-product for $(a)$ the dynamically perturbed case and $(b)$ the statically perturbed case.

velocity gradient should underestimate the dynamic extrema of the gradient and thus the scaling $u_{s}$ should be lower than necessary in this region; this is indeed the case, where the magnitude of underestimate is approximately given by the ratio of the r.m.s. to mean values of the gradient.

The successive profiles of the triple product $\sqrt[3]{{\overline{u^{\prime 3}}(y)}}$ show a negative region at $y=0.09 \delta=1.3 k_{r m s}$, but not the positive region at the roughness height that was observed in the statically perturbed case. The discrepancy maps (figure 7) highlight the difference between the dynamic and statically perturbed cases.

\subsection{Internal layers}

The internal layers, sketched in figure 1, represent the extent to which different boundary conditions have influenced the flow. The mean boundaries of the internal layers can be detected by three methods. The traditional methods, as outlined in Antonia \& Luxton (1971) and Andreopoulos \& Wood (1982), involve streamwise differentiation or rescaling of the mean velocity profile to locate stationary points which represent the edges of the internal layers (figure 8). Since the strength of the impulse $M$, noted above, is less for the dynamic case, it would be expected that the internal layers should grow more slowly, and indeed, both internal layers grow more slowly under the dynamic perturbation than under static perturbation.

In Jacobi \& McKeon (2011), two physically motivated methods were developed for locating the internal layers, both involving constructing discrepancy maps between the perturbed flow field and the unperturbed field. In one case, a map is constructed from the velocity scale related to the mean velocity gradient, $u_{s}$ (3.3) and this is shown for the present study in figure 9; in the other, a map is constructed from the third-order moment of the streamwise velocity signal. Both maps tend to identify the edge of the first internal layer quite easily for both dynamic and static perturbations. The second internal layer edge arguably appears in the $u_{s}$ map for the dynamic perturbation in the negative layer near the wall. But the third-moment map shows no trace of the second internal layer, and thus fails to provide a method of identifying the internal layers.

\subsection{Integral length scales}

Roughness is understood to affect the local scale sizes in a flow, so another approach to identifying regions of the flow field influenced by the roughness impulse is to look for regions in which scale sizes vary from the corresponding unperturbed flow, by 


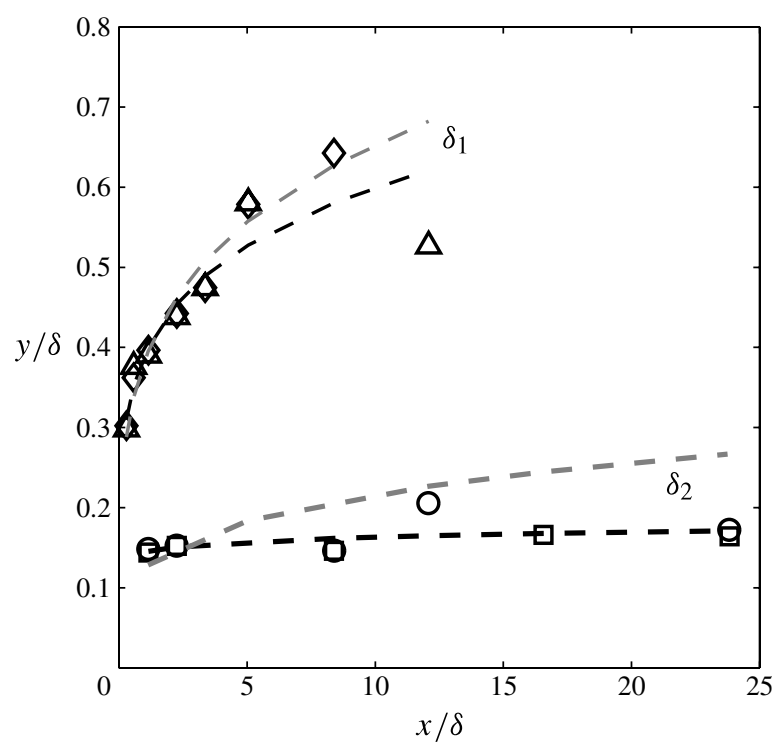

FIGURE 8 . The development of the internal layers, calculated by both methods described in the text. Plot and fit in outer units. For $\delta_{1}: \diamond$, via $y^{1 / 2}$-scaling; $\Delta$, via $\partial U / \partial x$. For $\delta_{2}: \bigcirc$, via $y^{1 / 2}$-scaling; $\square$, via $\partial U / \partial x$. Least-squares best fits:,$-- \delta_{1} / \delta=0.39(x / \delta)^{0.19}$; ,$-- \delta_{2} / \delta=0.14(x / \delta)^{0.05}$. Also included for reference, the best fits for the static impulse (in grey):,$-- \delta_{1} / \delta=0.38(x / \delta)^{0.23} ;--, \delta_{2} / \delta=0.12(x / \delta)^{0.24}$.

considering changes in the integral length scale $\Lambda_{L}$, as elaborated in Jacobi \& McKeon (2011), where $R_{11}$ is the autocorrelation of the streamwise velocity signal:

$$
\Lambda_{L}(x, y)=U(y) \int_{0}^{\infty} R_{11}(x, y, t) \mathrm{d} t .
$$

There is a second method for identifying the internal length scale, by using the streamwise spectrum

$$
\Lambda_{L}(x, y)=\frac{2}{\pi} \lim _{k_{x} \rightarrow 0} \phi_{x}\left(k_{x}\right) .
$$

and considering the limit as wavenumber approaches 0 . This method is attractive in general because it avoids a number of the difficulties associated with integrating the streamwise autocorrelation, as described in Builtjes (1975), but in particular, the contrast between the spectral and autocorrelation methods is insightful in the case of dynamic forcing.

The integral scale at each wall-normal and streamwise location reveals the relative distribution of the largest scales in the flow field, under the smooth and perturbed boundary conditions. A map of the ratio of these two sets of integral scales, perturbed normalized by unperturbed, determined by each calculation method for the dynamic perturbation is provided in figure $10(a, b)$. The spectral method shown in figure $10(a)$ identifies two distinct regions: one of increased integral scales, between the edges of the two internal layers, and one of decreased scales nearer to the wall. This same effect was seen also in the map of the statically perturbed flow, shown in figure 10(c). The variation of the integral length scales between both internal layers indicates that neither is in a state of equilibrium. When the calculation is conducted by 
(a)

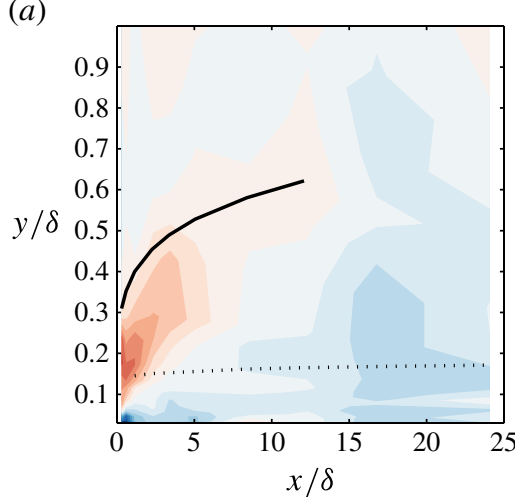

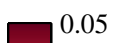

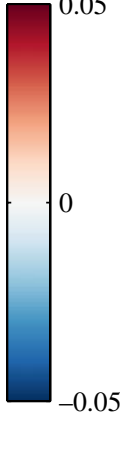

(b)

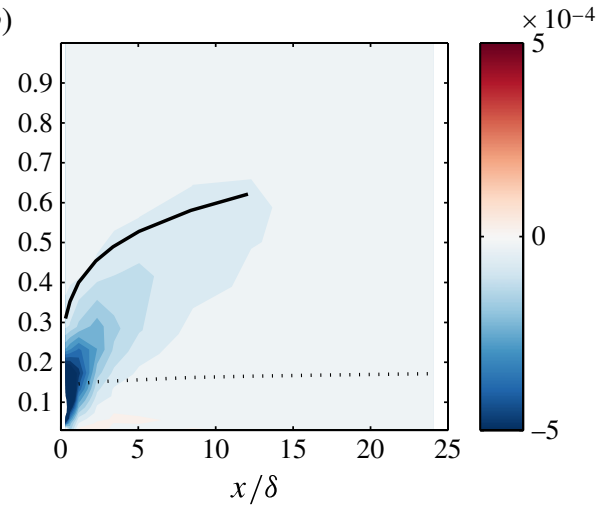

FIGURE 9. (a) The discrepancy maps for $u_{s}$ and (b) the map for the third-order moment of the streamwise velocity component. While the third-order moment map tended to highlight the precise inter-layer region for the case of a static perturbation, in the case of the dynamic perturbation it no longer serves as a useful criterion.
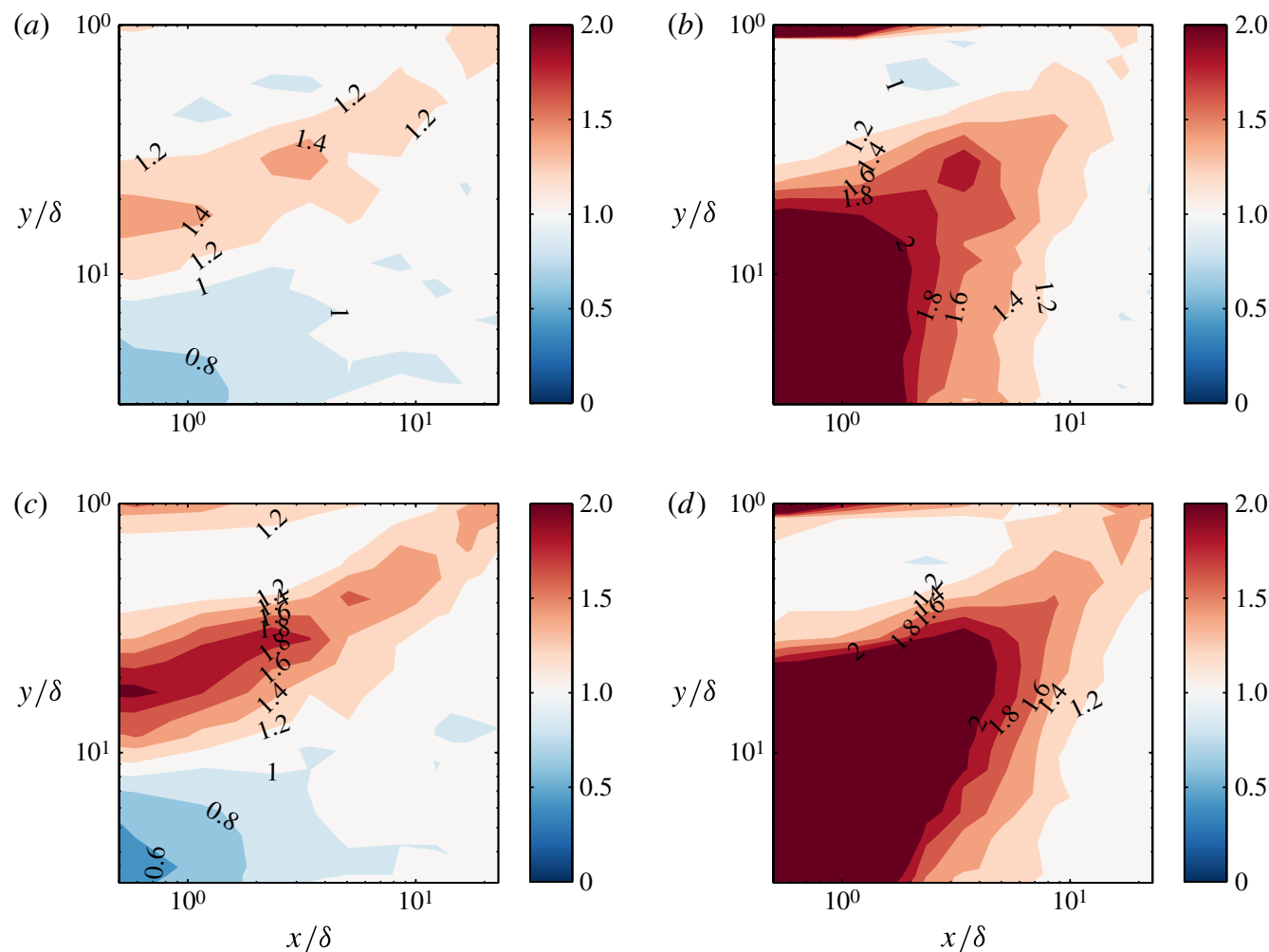

FIGURE 10. Top row, maps of the ratio of integral length scales between the dynamically perturbed flow and the unperturbed flow, calculated by the spectral method $(a)$ and integration of the autocorrelation $(b)$. Bottom row, maps of the ratio of integral length scales: for the statically perturbed flow calculated by the spectral method $(c)$ and for the dynamically perturbed flow calculated by a modification of the spectral method $(d)$, whereby the influence of the spectral peak due to the perturbation is accounted for in the limit. 
means of the autocorrelation, the resulting map, shown in figure 10(b) shows a large region of increased scales and no significant suppressed scales. In order to resolve the discrepancy, the map was re-calculated using the spectral approach, but this time, instead of extrapolating the flat portion of the spectrum from the very lowest measured wavenumber to produce the limit $k_{x} \rightarrow 0$, the limit was taken by extrapolating an average of the low-wavenumber spectral magnitudes which included the peak of the (low-wavenumber) input perturbation. The result, shown in figure $10(d)$, reproduces very closely the result from the autocorrelation.

The difference between the two spectral calculations is the inclusion or exclusion of the spectral peak associated with the input perturbation. When it is excluded by the limiting process, then the distribution of scales looks the same as the statically perturbed flow. But when it is included, by altering the limit such that the spectral peak is averaged into the region of the flat spectra at low wavenumbers, then the distribution of scales is significantly biased by the input of large scales - seen also in the autocorrelation method in figure 10(b), which includes this contribution. In other words, we see that the two contributions of the dynamic perturbation are separable in terms of the effect they have on the scales of the flow.

\subsection{Composite spectra}

In order to further investigate the impact of the dynamic perturbation on the structural composition of the flow field, the temporal spectra for each wall-normal and streamwise position were transformed by Taylor's hypothesis into spatial spectra in streamwise wavelength $\lambda_{x}$. Following the procedure outlined in Hutchins \& Marusic (2007), composite premultiplied spectra (in $\lambda_{x}$ and wall-normal position $y$ ) are reproduced for the unperturbed and statically perturbed flows of Jacobi \& McKeon (2011) for reference in figure 11. This procedure was then performed at all of the streamwise measurement locations downstream of the dynamic perturbation (figures 12 and 13). Key features of the turbulent boundary layer spectra, elaborated in Monty et al. (2009), were superimposed over the composite spectra in order to put the features of the perturbed flow in the spatial context of the accepted characteristics of the unperturbed spectra, namely: the inner peak at $\lambda_{x}^{+} \approx 1000, y^{+} \approx 20$; the peak for superstructures at $\lambda_{x} / \delta \approx 6$; and the large-scale motion (LSM) peak at $\lambda_{x} / \delta \approx 3$. Note, however, that the relatively low Reynolds number means that the latter two signatures are weak in the spectra shown here. The region of the mean recirculation bubble was estimated to extend to $x / \delta \lesssim 0.3$, based on the location of a significant change in the profile of $\sqrt{\overline{{u^{\prime 2}}^{2}(y)}}$.

The spectral composite maps for the dynamic perturbation share a few key trends in common with the static perturbation: in both, beyond the streamwise point at which the mean flow is unambiguously reattached downstream of the roughness, there is a clear suppression of the near-wall peak, particularly at large-wavelengths. In addition, for both perturbations, a large region of increased turbulent spectral intensity appears displaced from the wall. And as with the static perturbation, the dissipation of this region of displaced intensity appears to occur more slowly than the corresponding recovery of the near-wall peak. However, in addition to these common features, the dynamic perturbation is distinguished by a spectral signature of the initial perturbation which persists throughout the entire boundary layer and throughout all of the streamwise measurement locations, as shown in figures 12 and 13. The presence of this spectral signature of the dynamic perturbation, even at the last measurement location, more than $20 \delta_{0}$ downstream, indicates that the flow field remains distinctively perturbed even after other statistical measures of the flow's 

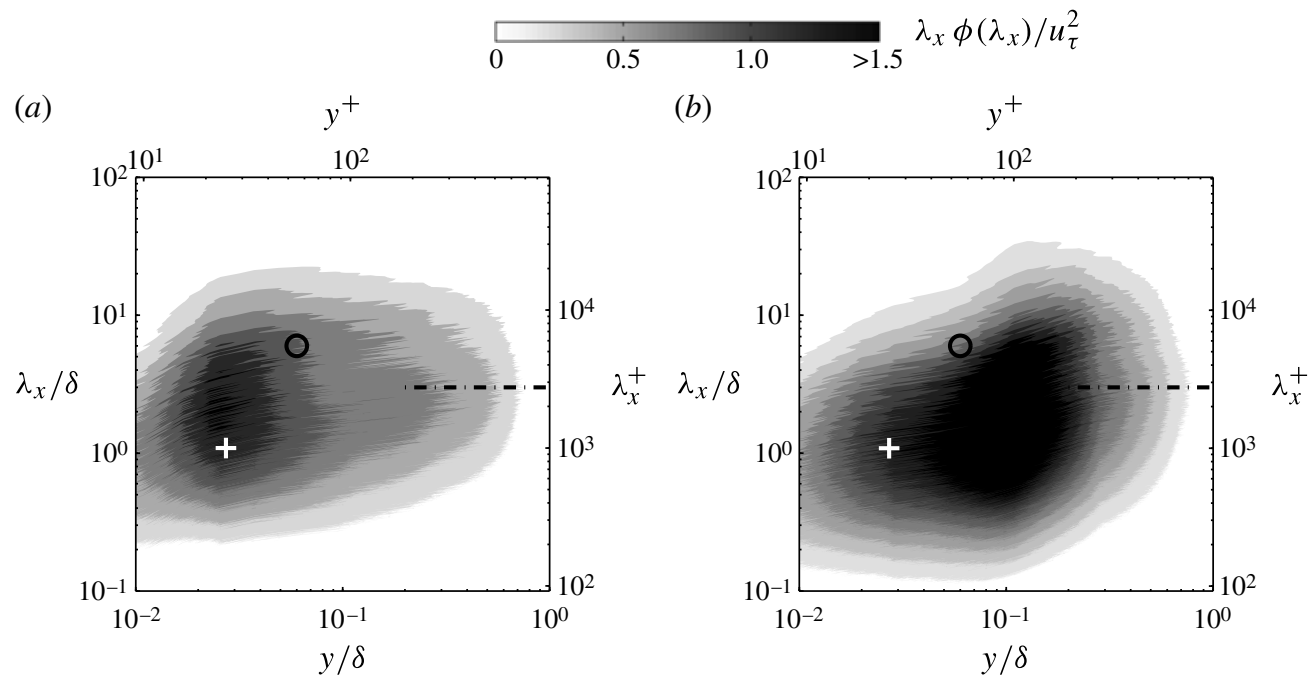

FIgURE 11. Composite spectra at $x / \delta=0.6:(a)$, Unperturbed $\operatorname{Re}_{\theta}=2770,(b)$ statically perturbed. The white cross marks the vicinity of the inner peak $\left(\lambda_{x}^{+} \approx 1000, y^{+} \approx 25\right)$, the black circle marks the expected location of the VLSM (very large-scale motion) peak at $\left(\lambda_{x} / \delta \approx 6\right)$, and -.- marks the peak along $\lambda_{x} / \delta \approx 3$.

relaxation show an approximate return to equilibrium. The periodic signature's vast extent also demonstrates the ability of even a localized impulsive perturbation to affect the entire downstream flow field, even far from the wall. Moreover, the choice of forcing frequency was selected in order to force precisely the range where the superstructure peak is expected at high Reynolds number (which is not clearly defined in the unperturbed flow at least until far downstream, where $R e_{\theta}=4040$ ).

Columns $(b)$ of figures 12 and 13 show discrepancy plots of the composite spectra, similar to those formed for the statistical quantities above. As mentioned in Jacobi \& McKeon (2011) there is an additional subtlety that the wavelength spectra between the perturbed and unperturbed flows vary as a consequence of the use of Taylor's hypothesis. In order to subtract equivalent ranges, the unperturbed composite spectrum was re-gridded (by cubic interpolation) to the range of the perturbed spectrum, prior to the subtraction. The unperturbed composite spectra were reasonably robust in the streamwise direction thus making this sort of subtraction justifiable, at least for qualitative observations. The discrepancy maps highlight both the imprint of the organized wave, as well as the partial suppression and recovery of the signature of the near-wall cycle discussed above.

\subsection{Decomposition of the turbulence intensity by spectral contribution}

The integral scale map in figure 10(a) showed suppressed scales near the wall, and by comparison with the statically perturbed flow it was shown that this suppression is a consequence of the spatial impulse aspect of the perturbation. On the other hand, the integral scale map in figure $10(b)$ (along with the composite spectra in figure 12) demonstrated that the organized wave aspect of the perturbation contributed long structures, which actually supplemented the loss of these same-sized structures due to the displacement effect. This same two-part effect was seen also in the $\sqrt{\overline{u^{\prime 2}(y)}}$ statistics. The profile for the dynamic perturbation showed a broader hump which encompassed the wall-normal locations of the static perturbation and extended 

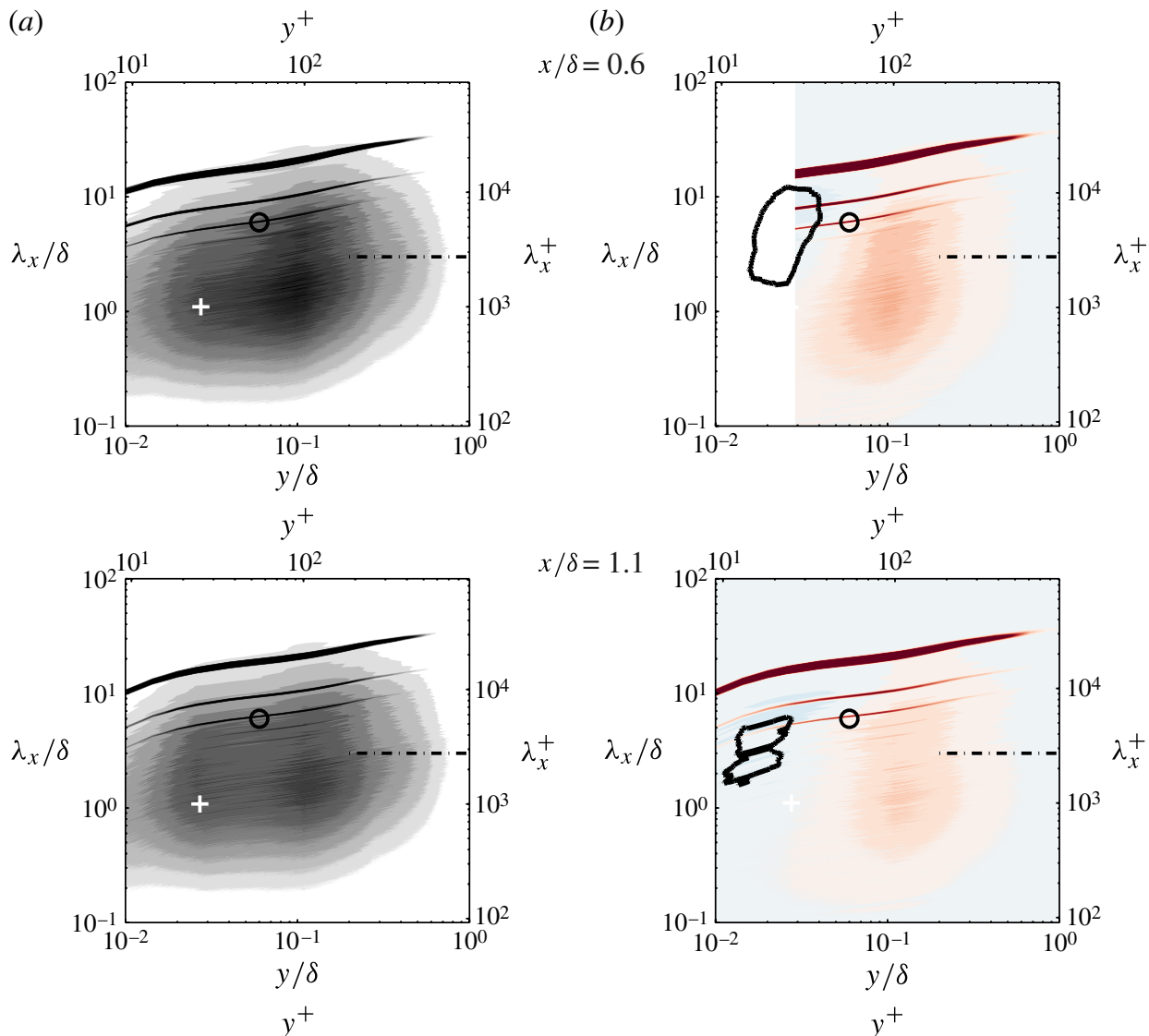

$$
x / \delta=1.1
$$
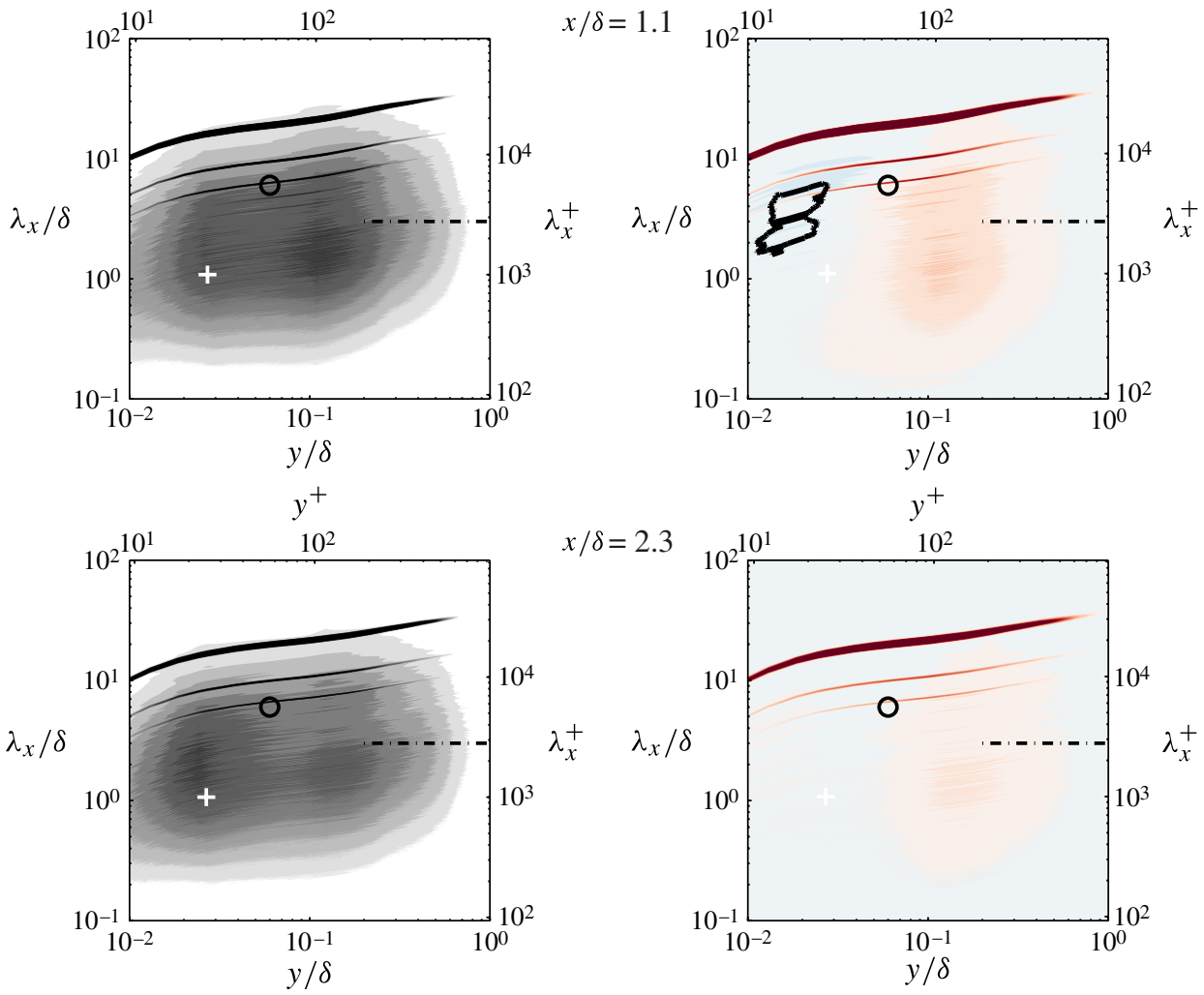

FIGURE 12. (Colour online available at journals.cambridge.org/flm) (a) Composite spectra for the perturbed case: the first streamwise location, $x=0.1 \delta=1.65 k$, is suspected to be within the mean recirculation bubble downstream of the last roughness element; therefore the third streamwise location $x=0.6 \delta=10 k$, which appears to be downstream of the recirculation region, is shown first. Levels follow figure 11 and are the same as in Jacobi \& McKeon (2011). (b) The discrepancy maps for the composite spectra, with a range identical to the spectra themselves, but mirrored for negative values (red are positive, blue negative, and are outlined by the contour line, which represents a region of spectral content suppressed more than $5 \%$ below the unperturbed flow). The dark bands, starting at the forcing frequency, represent the input forcing and its associated harmonics. 
(a)
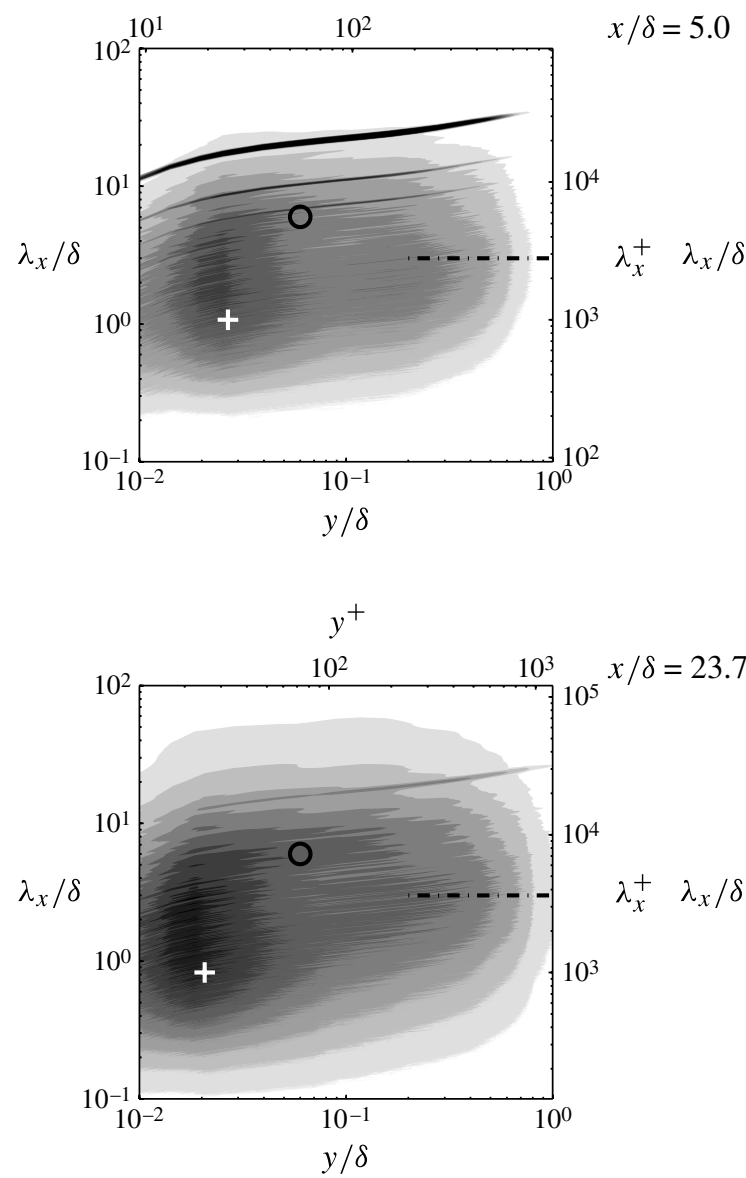

(b)

$x / \delta=5.0$

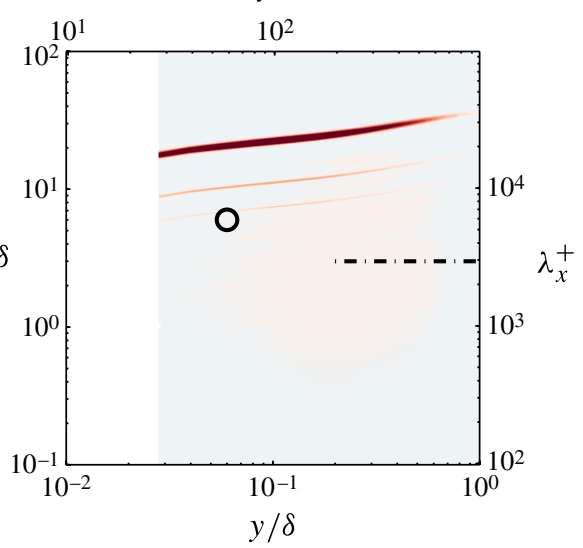

FIgURE 13. (Colour online) (a) Composite spectra continued from figure 12 at additional downstream positions. (b) Continuation of the discrepancy maps for the composite spectra.

far closer to the wall. That hump appears in the composite spectra as the broad energetic region, farther from the wall than the near-wall peak, but in approximately the same region of wavelength space. Since the two different features of the dynamic perturbation - the organized wave and the impulsive roughness - manifest themselves distinctly from a spectral perspective, it seems reasonable to consider exploiting this spectral distinction in order to disentangle the statistical picture. Since the spectra at each wall-normal location are normalized by the corresponding values of $\sqrt{\overline{{u^{\prime 2}}^{2}(y)}}$,

$$
\overline{u^{\prime 2}}(x, y)=\int_{\lambda_{x}=\lambda_{a}}^{\lambda_{x}=\lambda_{b}} \Phi\left(\lambda_{x}^{\prime}, x, y\right) \mathrm{d} \lambda_{x}^{\prime},
$$

a decomposition of the $\sqrt{\overline{u^{\prime 2}(y)}}$ profiles by spectral contribution is possible, in order to isolate the particular influence of the dynamic perturbation above and beyond the static perturbation.

Now, if structures associated with the impulsive static roughness are presumed significantly smaller than those associated with the dynamic perturbation, which can 
be inferred from the dynamic composite spectra (figure 12), then the wavelengthspace of the composite spectra can be partitioned into large and small wavelengths, corresponding to the distinctive contributions from the static and dynamic parts of the perturbation. The composite spectra are integrated over bands of wavelengths $\left(\lambda_{a} \rightarrow \lambda_{b}\right)$ in order to reproduce the previous discrepancy contour plots of figure 4 , but this time including contributions to $\overline{\left(u^{\prime 2}\right)}(x, y)$ from only distinct wavelength bands (figure 14). The division between large and small wavelengths was set at $7 \delta$ in order to include the superstructure peak $(\approx 6 \delta)$ on the 'small-wavelength' side of the division, which allows for separating the artificially injected, longer wavelengths more easily.

A line has been fitted by least-squares regression to the peaks of the deviation, $\Delta \overline{u^{\prime 2}}(x, y)$, of the perturbed $\overline{u^{\prime 2}}(x, y)$ from that of the unperturbed flow. This line shows that a power-law relation describes the shift of the hump associated with the perturbations, as the hump shifts away from the wall and as it decreases in magnitude moving downstream. However, the rate at which this shift occurs is observed to be quite different between the static and dynamic perturbation, as seen in the difference in the inclination of the propagation of the hump between left and right columns. The consequence of this difference is that the hump associated with the static perturbation is expected, ignoring mixing and other effects downstream, to clear the boundary layer $75 \delta$ downstream of the impulse (extrapolating the power law to $y / \delta=1$ ), whereas the hump associated with the dynamic perturbation would clear the boundary layer in $50 \delta$.

However, if the contour plot of the deviation is recalculated according to the above procedure for only small-wavelength contributions to $\overline{u^{\prime 2}}(x, y)$, then the static and dynamic cases appear identical. And since, for the static case, this particular range is assumed to relate to near-wall turbulent motions which were displaced over the two-dimensional roughness, as well as any shedding from the elements, it could be inferred that this range also describes similar behaviour in the dynamic case. It is observed further that the structures associated with this range move away from the wall at an identical rate in the static and dynamic cases. Whereas, when the contours are recalculated based on contributions from large wavelengths - the kind associated with the dynamic perturbation - the shift of the associated hump away from the wall occurs at detectably different rates in the two cases, in addition to the difference in amplitudes.

In some sense, the hump from the dynamic perturbation has been decomposed into a contribution which behaves like the hump seen in the statically perturbed case, and an additional energetic content from the dynamic aspect, and these two distinct contributions behave differently both in spatial extent in the flow field and in the rate at which they evolve downstream. The common features include the effect on the spectral signature of the near-wall cycle, the stress bore, and the internal layers. However, the particular contribution from the dynamic case, in the form of the structured (organized) addition of energy to the flow, manifests itself in the redistribution of scales in the flow. The caveat to this proposed decomposition is that, as indicated by the harmonics visible in the spectra of figures 12 and 13, nonlinear dynamics are certainly present. In order to bolster the claim of separability then, a phase-locked decomposition of the velocity signals is considered in the following sections in order to demonstrate that, at least for practical engineering of the flow field, the linear interactions are the most significant. 
(a)

(b)

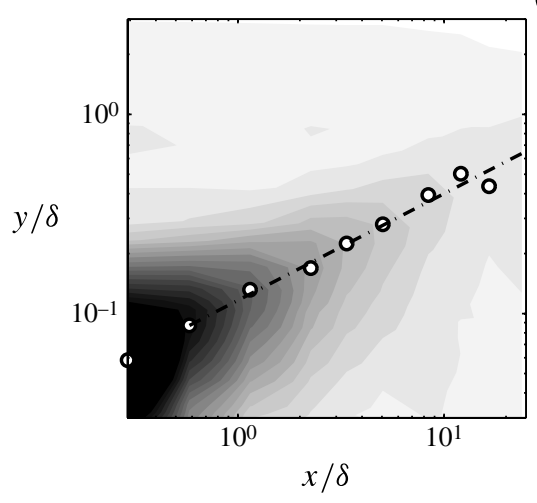

$0<\lambda / \delta<\infty$

$y / \delta=0.12(x / \delta)^{0.54}$

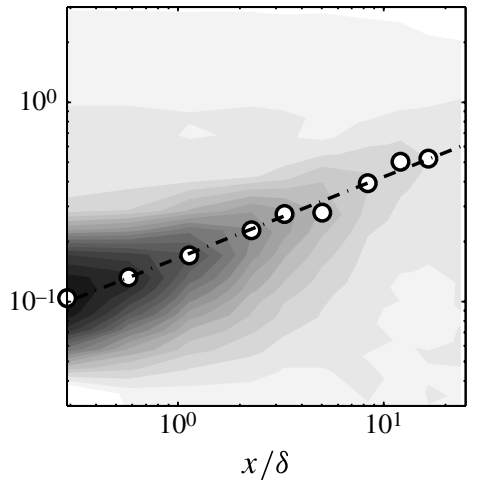

$0<\lambda / \delta<7 \quad y / \delta=0.17(x / \delta)^{0.41}$
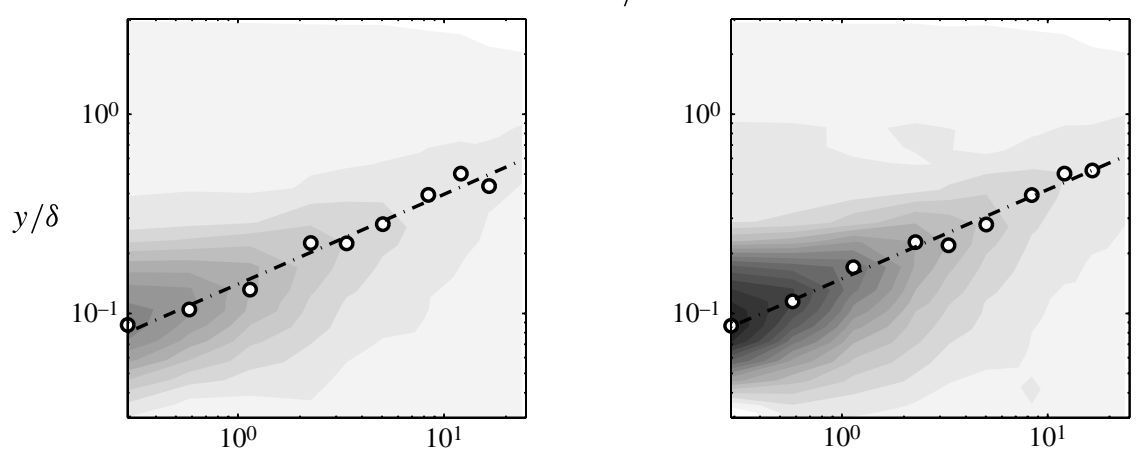

$x / \delta$

$y / \delta=0.14(x / \delta)^{0.45}$

$x / \delta$

$7<\lambda / \delta<\infty \quad y / \delta=0.15(x / \delta)^{0.45}$
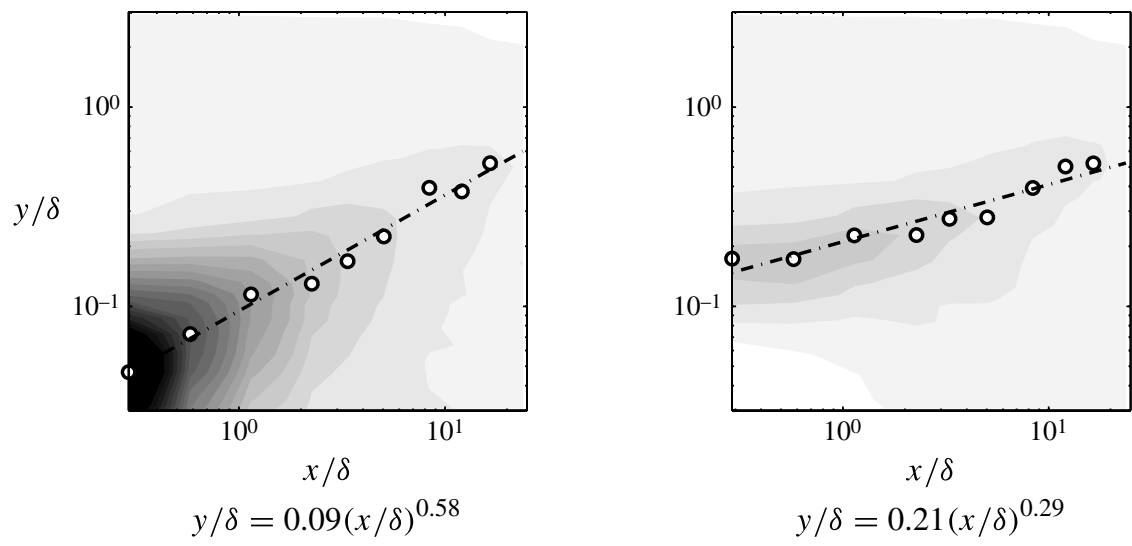

FIGURE 14. Recreating the turbulence intensity variation (figure 4) by integrating the streamwise wavelength spectra over different ranges of wavelengths: in the top row, the entire wavelength range is integrated to reproduce the previous result, and the second and third rows show short and long wavelength contributions separately. (a) The contour map for the variation of the dynamic impulse from the smooth case; $(b)$ the variation of the static impulse from the smooth. $\bigcirc$, the trace of the peaks; ---, the power-law fits for the peaks, expressions for which are given below each panel; all levels are the same as in figure 4 . 


\section{The dynamic perturbation as an organized wave}

Having established that the effect of the dynamic perturbation appears to manifest itself in two different ways: as an impulsive roughness effect and as a coherent and persistent organized wave, a phase-locked decomposition following Hussain \& Reynolds (1970) is now developed and key flow properties are examined within that context. In particular, distinct fluctuating velocity modes are identified, and it is shown that they present the classic features of critical-layer organized waves. The properties of the wave are measured and a modified Orr-Sommerfeld operator is employed to predict the observed mode-shapes. The Orr-Sommerfeld analysis involves a linearization of the Navier-Stokes equation (NSE); this will be justified in the present case following McKeon \& Sharma (2010), since the amplification of the input disturbance (forcing) is significant enough to be considered dominant in the following resolvent analysis of the Orr-Sommerfeld equation.

\subsection{Phase-locked decomposition}

Considering the standard form of the phase-locked decomposition, used by Hussain \& Reynolds (1970), and reported in (2.2), the fluctuation from the Reynolds decomposition $u^{\prime}(y, t)$ is further divided into a contribution from the periodic perturbation, $\tilde{u}(y, t)$, and a fluctuation about that periodic contribution $u_{t}^{\prime}(y, t) . \tilde{u}(y, t)$ is calculated from the phase average of the velocity signal, $\langle u(y, t)\rangle$. The phase average is obtained by first dividing the period of the disturbance into $n$ segments, then sampling the velocity signal at each segment and finally averaging across segments so that the angle-brackets represent an ensemble average over phases. $\tilde{u}(y, t)$ is then the difference between the mean value, $U(y)$, and the phase-averaged value, $\langle u(y, t)\rangle$, and itself has, by definition, zero mean.

By combining the measurements of $\tilde{u}(y, t)$ at all of the wall-normal locations, maps of the wall-normal variation of $\tilde{u}(y, t)$ over a mean period (written as $[0,2 \pi]$, as determined from the experimental frequency) can be generated for each streamwise measurement location, as shown in figure 15. In all of the following phase-locked maps, the contour lines (with levels at intervals of $20 \%$ of the maximum) indicate contours scaled on the streamwise-local amplitudes, whereas the colour levels are scaled to be consistent across all streamwise positions to allow comparison of relative amplitudes. The persistence of the distinctive shapes of the variation in the decomposed velocity signals, visible via the contour lines even at the most downstream measurement location, testifies to the strong coherence of the stress wave and to the fact that it is a local phenomenon, independent of the fact that the adjacent wall condition is smooth. Also, the periods are plotted to reflect the physical phase shift moving downstream, from which the wavenumber of the perturbation is inferred below.

The contours of the variations in $\tilde{u}(y, t)$ highlight both the inclination of each mode to the wall and its concentration relatively close to the wall (most prominently for $y / \delta<0.2$ ). The colours (indicating the sign and magnitude of each variation) show a phase shift of $180^{\circ}$ in the wall-normal direction, although this phase shift is most obvious only for the first five streamwise locations. Importantly, the location of the maximum amplitude tends to drift away from the wall moving downstream, and since the plots are shown in outer units, the rate of this drift is faster than the boundary layer growth. The modes appear to have a shallow downstream inclination for small wall-normal distances, but then lean upstream further from the wall.

In order to obtain the maps for the wall-normal velocity component, a similar phase-locking analysis was conducted on the PIV data. The nature of the velocity 

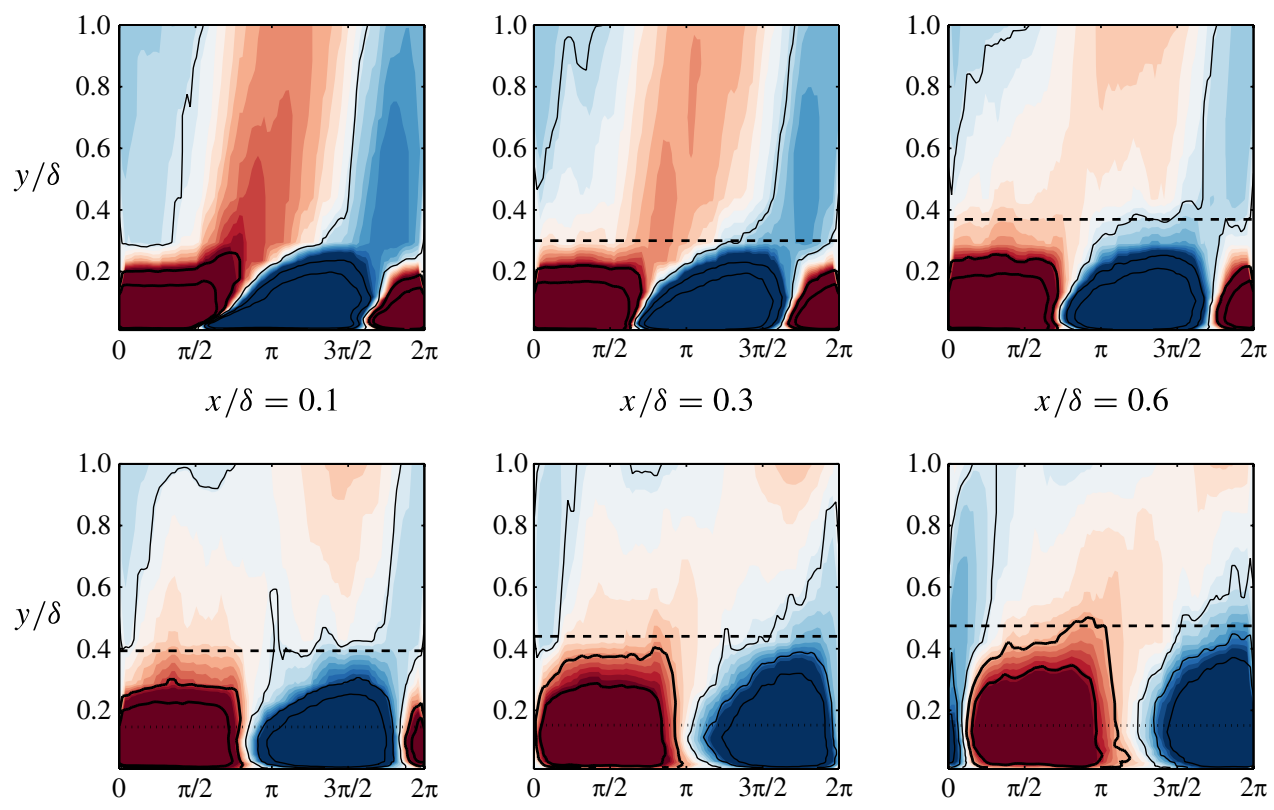

$x / \delta=1.1$

$$
x / \delta=2.3
$$
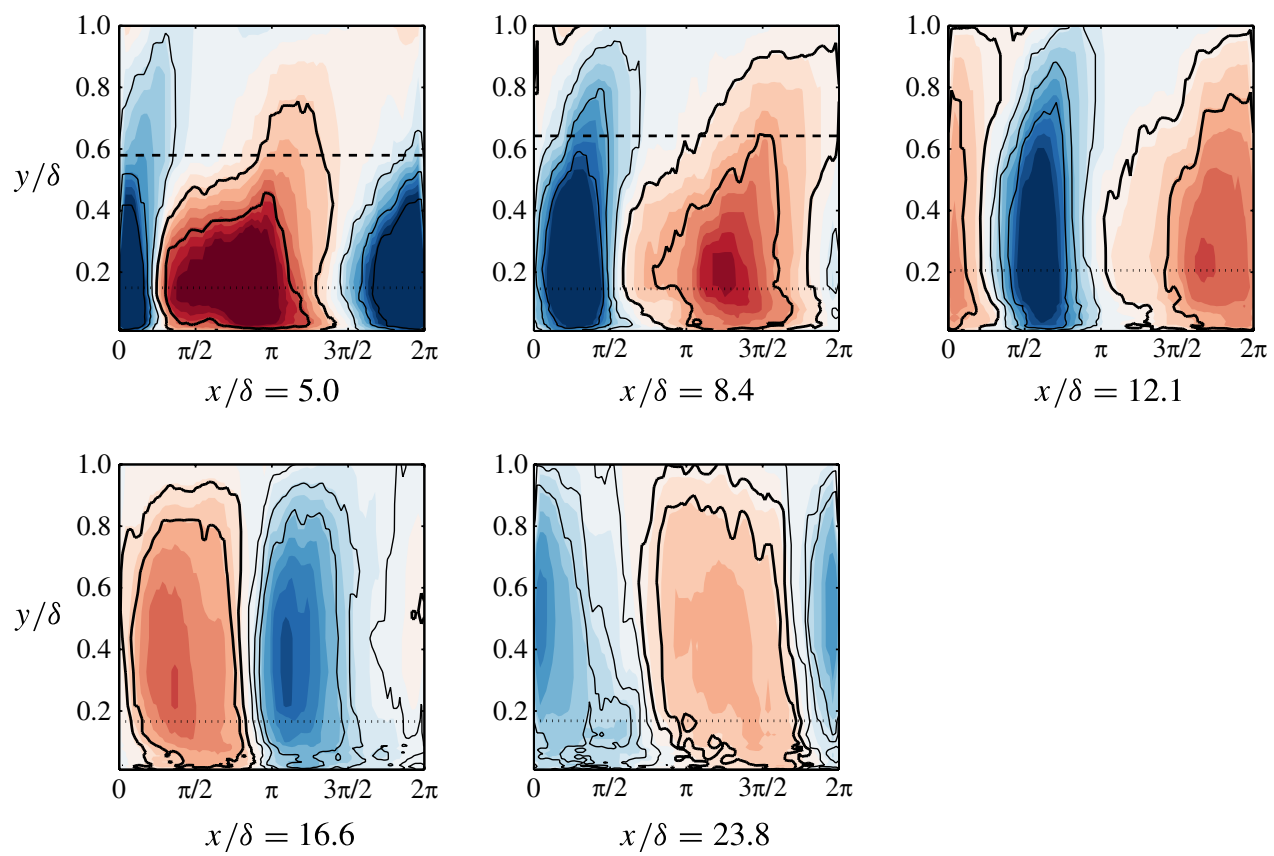

FIGURE 15. A map of $\tilde{u}(y, t)$ over an average period (abscissa $t \in[0, \pi]$ ) in outer units (ordinate $y / \delta$ ) at all of the streamwise locations. The colour levels are fixed for all plots, so the amplitude of the mode is physically represented, but the contour lines are scaled per streamwise location and thus represent the shape independent of amplitude. The mean internal layer locations, when interpolatable, are denoted: - - first internal layer, and ..., second internal layer. 
decomposition for the case of the PIV is somewhat different due to the additional streamwise variation within the PIV field. The PIV fields were first averaged in the streamwise direction, over $x$ and then the decomposition described above was applied

$$
v(x, y, t)=V(y)+\tilde{v}(y, t)+v_{1}^{\prime}(y, t)+v_{2}^{\prime}(x, y, t) \approx V(y)+\tilde{v}(y, t)+v_{t}^{\prime}(y, t),
$$

such that there are in fact two turbulent contributions: $v_{1}^{\prime}(y, t)$ is the fluctuation about the streamwise-averaged, phase-averaged field, and $v_{2}^{\prime}(x, y, t)$ is the fluctuation about the phase-averaged field due to the variation in the $x$-domain: For computational simplicity, these two separate turbulent contributions were treated together, such that the $v_{t}^{\prime}(y, t)$ from an extension of (2.1) to the wall-normal component is equivalent to $v_{1}^{\prime}(y, t)+v_{2}^{\prime}(y, t)$ under the present analysis. The PIV phase-locking was also accomplished somewhat informally, without an external reference, by comparison with the velocity signal nearest to the wall, where the signal was well preserved and strong enough to allow for a reliable phase-lock as shown by validation against the formally phase-locked hot-wire analysis. The wall-normal phase-locked maps are reproduced in $\S 5.2$ (figure 24). The wall-normal contours show more elongated shapes, not attached closely to the wall like the streamwise modes, and they also contrast with the streamwise modes in their lack of prominent inclination and $180^{\circ}$ phase-shift. The mean amplitude of the streamwise modes was $\approx 2$ times the magnitude of the wall-normal modes, whereas the peak of the streamwise mode was roughly an order of magnitude larger than the wall-normal mode peak $(\approx 5.7)$.

The significance of the fluctuating quantities $u_{t}^{\prime}(y, t)$ and $v_{t}^{\prime}(y, t)$ and their relationship to the periodically changing quantities are the subject of ongoing analysis and will be presented in future work.

\subsection{Experimental parameters of the perturbation}

Having observed the output of the dynamic perturbation in the form of distinct modal shapes in both velocity components, the question of the precise nature of the input remains. While the input frequency can be set externally and the roughness is essentially two-dimensional, the flow effectively sets the streamwise wavelength through the length scale associated with flow separation and reattachment either side of the roughness perturbation. However, the use of multiple, phase-locked, streamwise measurements provides a straightforward means of inferring the wavenumber of the dynamic perturbation from the streamwise development of the amplitude and phase of the modes. The detailed calculations are described extensively in Hussain \& Reynolds $(1970,1972)$ and a brief overview is provided below.

The real part of the wavenumber $k_{r}$ is inferred from the rate of change of the phase of the perturbation (denoted by the angle symbol, $\angle$ ) with streamwise distance, $k_{r}=\partial(\llcorner\tilde{u}) / \partial(x / \delta)$. This relationship is linear for most of the downstream extent of the perturbation, and therefore the slope is obtained by least-squares fitting to a line,

$$
\angle \tilde{u}=0.336(x / \delta)+1.040 .
$$

However, in the immediate vicinity of the roughness perturbation, for $x / \delta \lesssim 1$, the rate of change is faster, and is described with a logarithmic fit

$$
\angle \tilde{u}=63.5 \log _{10}(x / \delta)+61.0 .
$$

The faster growth rate is a consequence of the velocity deficit (or region of reversed flow) immediately downstream of the roughness. As this deficit quickly recovers, the rapid change in the mean velocity profile results in a nonlinear phase change over a very short distance. Previous studies by Hussain \& Reynolds (1972) considered 

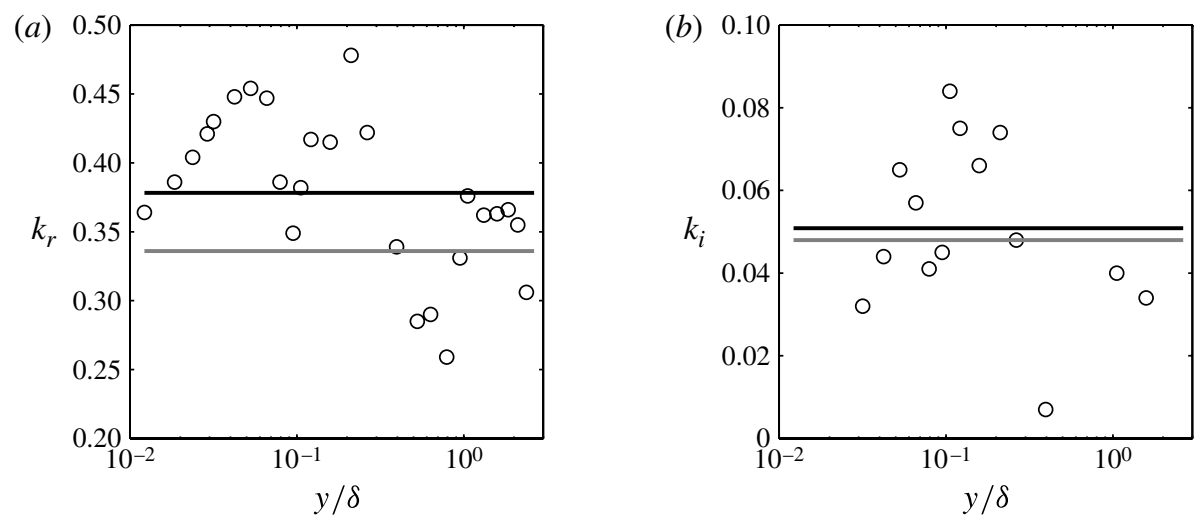

FIGURE 16. The variation in the measurement of values of the streamwise wavenumber for the perturbation, $k=k_{r}+\mathrm{i} k_{i}$ when calculated as described in the text at different wall-normal locations. (a) The variation in $k_{r}$ (circles); mean $k_{r}=0.378$ (black line); calculated from the peak signal strength, $k_{r}=0.336$ (grey line). (b) The variation in $k_{i}$ (circles). Mean $k_{i}=0.051$ (black line); calculated from the peak signal strength, $k_{i}=0.048$ (grey line).

only locations sufficiently far from the input for the linear growth rate to be obtained. By considering closer points also, the phase-shift can be used as a second confirmation that the measurements beyond $x / \delta \approx 1$ are well clear of any significant flow reversal; however this estimate is quite conservative, since it also includes significantly decelerated, but not reversed, flow and thus is consistent with the previous estimate of the recirculation bubble itself for $x / \delta<0.3$. The important consequence of this is that the wavespeed inferred from the linear fit applies only to the region where the linear fit is itself valid.

The streamwise variation was measured at the wall-normal locations where the wave amplitude was largest. However, the same process could be carried out at all of the wall-normal measurement locations, conducting a series of streamwise comparisons for each wall-normal location (in outer units). Using this method results in a significant amount of variation, as shown in figure 16. Following a similar method, the imaginary component of the wavenumber can also be inferred and is shown; this quantity will be discussed below. It is clear, however, from figure 16 that the error is not random, but rather is strongly biased by the wall-normal location of the measurements, with higher magnitudes of both wavenumbers in the inner region of the boundary layer outside the buffer layer, and decreasing magnitude moving towards the wall or towards the intermittent edge of the boundary layer. To understand this discrepancy between the two methods of calculating the wavenumbers, the ratio of the real component of the wavespeed to the mean convective velocity is plotted in figure 17 , where the complex wavespeed is defined as $c=\omega / k=c_{r}+\mathrm{i} c_{i}, \omega$ being the input frequency. The fact that the wavespeed measured as a function of wall-normal location $c_{r}(y)$ is smaller than the wavespeed at the location of maximum wave amplitude $c_{r}$ across most of the boundary layer (except in the intermittent region) is a consequence of the observation that the location of the maximum wave amplitude drifts away from the wall faster than the rate of boundary layer growth, as reported above. In other words, over a fixed streamwise distance, the peak perturbation has travelled further away from the wall and thereby advanced further in phase than it would have if it remained at a fixed height in outer units. 


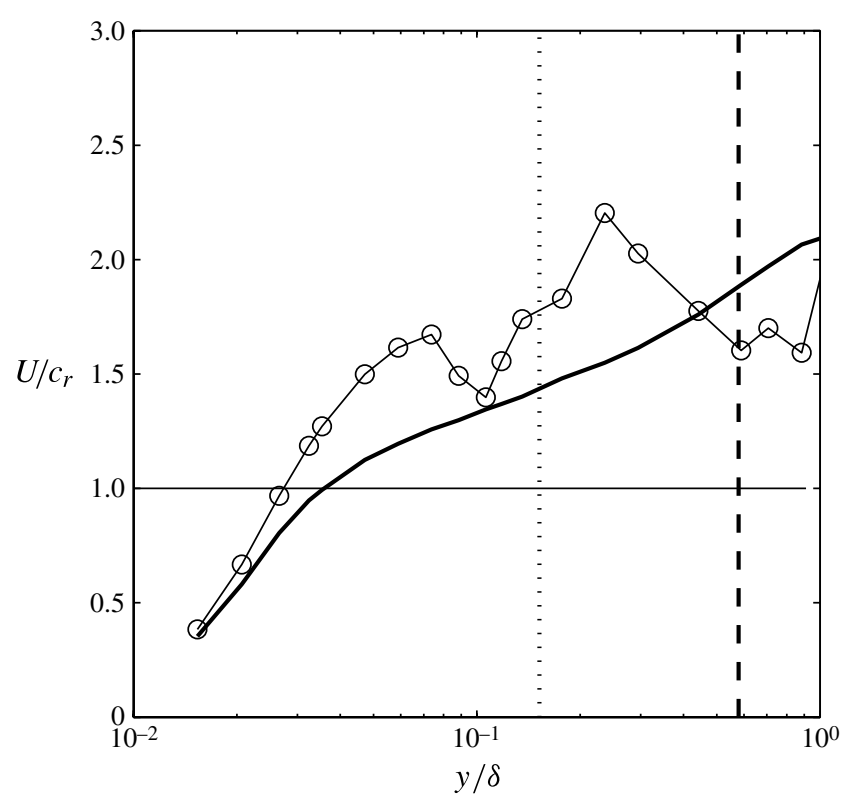

FIGURE 17. The mean unperturbed convective velocity $U(y / \delta)$ divided by the real part of the wavespeed $c_{r}(y / \delta)$ which was calculated from the gradient of the phase change at each wall-normal position $(\bigcirc)$. The same ratio of speed, but with the real part of the wavespeed $c_{r}$ calculated from the gradient of the change in phase at the location of the peak perturbation strength (-), showing a crossing at $y_{c} / \delta=0.04$, which indicates the presence of a critical layer. The mean location of the internal layers, identified above in figure 8 , have been marked for the streamwise component: --- , the first internal layer; $\cdots$, the second internal layer.

Calculating the wavenumber at the location of maximum amplitude yields a wavespeed of $c=0.464-0.066 \mathrm{i}$ in outer units, which means that the disturbance is not strictly 'neutral', but rather decays, consistent with the results of Hussain \& Reynolds (1972). The wavelength for the dynamic perturbation appears to be quite long - constituting approximately $17 \%$ of the total length of the plate or a little more than a third of the section downstream of the perturbation. The key parameters for the perturbation are provided in table 2 (along with additional comparisons, relevant to the subsequent analysis), where all terms are non-dimensionalized in outer units, except the frequency of the perturbation, $f$, which is left dimensional for comparison. Note that the equivalence of the wavespeed with the local mean velocity, $u / c_{r}=1$, which occurs in the vicinity of $y / \delta=0.04$ is a characteristic of a 'critical' disturbance, as will be discussed further below.

The amplitude of the perturbation is also of significant interest. In particular, the ability of a single mode or small number of modes of the resolvent analysis to accurately represent the observed dynamics is expected to depend on the relative strength of the 'external forcing' in relation to the 'internal forcing'. As noted in $\S 1$, the forcing considered by Hussain \& Reynolds (1970) was actually weaker, in one sense, than that achieved by the ribbon in early transition studies of laminar flow, shown in table 3, presumably making the signature of the perturbation difficult to discriminate from other influences in the flow. The current experiment, by virtue of the roughness perturbation, generates a stronger perturbation, thereby increasing the 


\begin{tabular}{lccccccc}
\hline & $k_{r}$ & $k_{i}$ & $\begin{array}{c}R e \\
=U \delta / v\end{array}$ & $\begin{array}{c}f \\
(\mathrm{~Hz})\end{array}$ & $\begin{array}{c}\omega_{r} \\
=2 \pi f \delta / U\end{array}$ & $\begin{array}{c}\lambda \\
=2 \pi / k_{r}\end{array}$ & $y_{c}$ \\
& & & & & & \\
& 0.336 & 0.048 & $2.24 \times 10^{4}$ & 30 & 0.159 & 18.70 & $0.04^{\mathrm{a}}$ \\
Experiment & 0.466 & 0 & $2.62 \times 10^{3}$ & & 0.163 & 13.48 & 0.209 \\
$\begin{array}{l}\text { Schlichting (1950) } \\
\text { Schubauer \& }\end{array}$ & 0.528 & & $2.64 \times 10^{3}$ & 103 & 0.161 & 11.90 & 0.2 \\
$\begin{array}{l}\text { Skramstad (1943) } \\
\text { Hussain \& }\end{array}$ & 0.981 & 0.073 & $1.38 \times 10^{4}$ & 25 & 0.746 & 6.40 & - \\
Reynolds (1970) & & & & & & &
\end{tabular}

TABLE 2. Parameters for the perturbation. ${ }^{\text {a }}$ Value at $x / \delta=0.1$; see discussion in the text.

Experiment

Schubauer \& Skramstad (1943)

Hussain \& Reynolds (1970)

$\begin{array}{cc}|\tilde{u}| / U & |\tilde{u}| / \sqrt{\overline{{u^{\prime 2}}^{2}}} \\ 2.5 \times 10^{-2} & 0.325 \\ 1.7 \times 10^{-2} & \\ 5.8 \times 10^{-3} & 0.059\end{array}$

TABLE 3. Magnitude of perturbation.

likelihood that a small number of modes of the resolvent should adequately describe the flow.

\subsection{Streamwise velocity mode development}

The qualitative observations regarding the shape of the modes, shown in figure 15, can be made more precise by calculation of the wall-normal profiles of the amplitude (figure 18) and phase (figure 19) of each mode, as they vary throughout the boundary layer, where the modes were picked out by means of a Fourier transform of the instantaneous velocity signal. This procedure was carried out for all of the PIV measurements taken at a single streamwise location, as well as the full range of streamwise locations interrogated with the hot-wire.

The variation of the amplitude of the streamwise mode shows a reasonably sharp peak near the wall, followed by a decay. The peak for the mean of the PIV runs is located at $y / \delta=0.13$; the mean for the hot-wire profiles is in roughly the same location, although there is significant variation in peak location with streamwise location. But in general, the variation in amplitude and phase for both velocity components substantiates the qualitative description offered above for a closely attached streamwise mode with a sharp phase change and a larger unattached wallnormal mode with minimal phase variation.

The streamwise variation was also identified for the location of the $180^{\circ}$ phase shift in $\tilde{u}(y, t)$, normalizing the initial value of the streamwise mode's phase, at the wall, to 0 for all streamwise locations. Both streamwise trends (figure 20) indicate that the mode associated with $\tilde{u}(y, t)$ is growing downstream of the perturbation, slowly detaching from the wall and enlarging the domain of the in-phase signal.

Having carefully described the precise behaviour of the modes which are exhibited under the dynamic perturbation, the challenge remains to predict them. McKeon \& Sharma (2010) proposed that turbulent wall-bounded flow can be modelled as a superposition of propagating modes of velocity fluctuations. Under such a framework, the mode observed as a result of the dynamic perturbation, above, is just a particular 

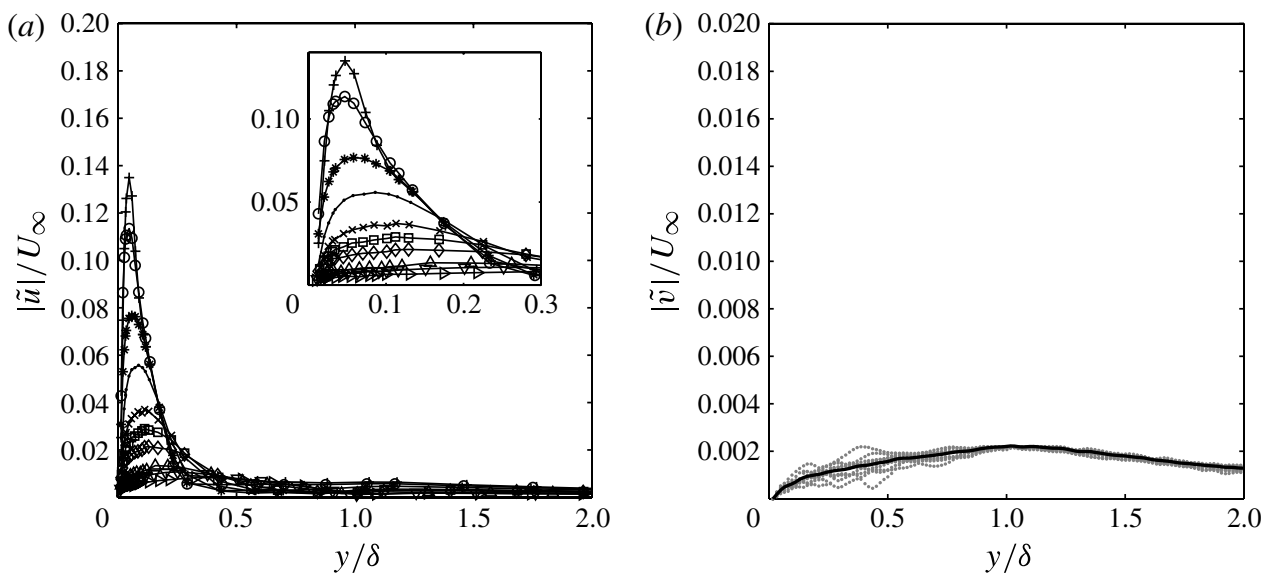

FIGURE 18. (a) The amplitude variation in $\tilde{u}(y, t) / U_{\infty}:+, x / \delta=0.1 ; \bigcirc, 0.3 ; *, 0.6 ; \cdot, 1.1$; $\times, 2.3 ; \square, 3.4 ; \diamond, 5.0 ; \triangle, 8.4 ; \nabla, 12.1 ; \triangleright, 16.6 ; \triangleleft, 23.8$. (b) $\tilde{v}(y, t) / U_{\infty}$ : light symbols refer to different PIV runs in order to indicate variability; bold line is the mean for all PIV.
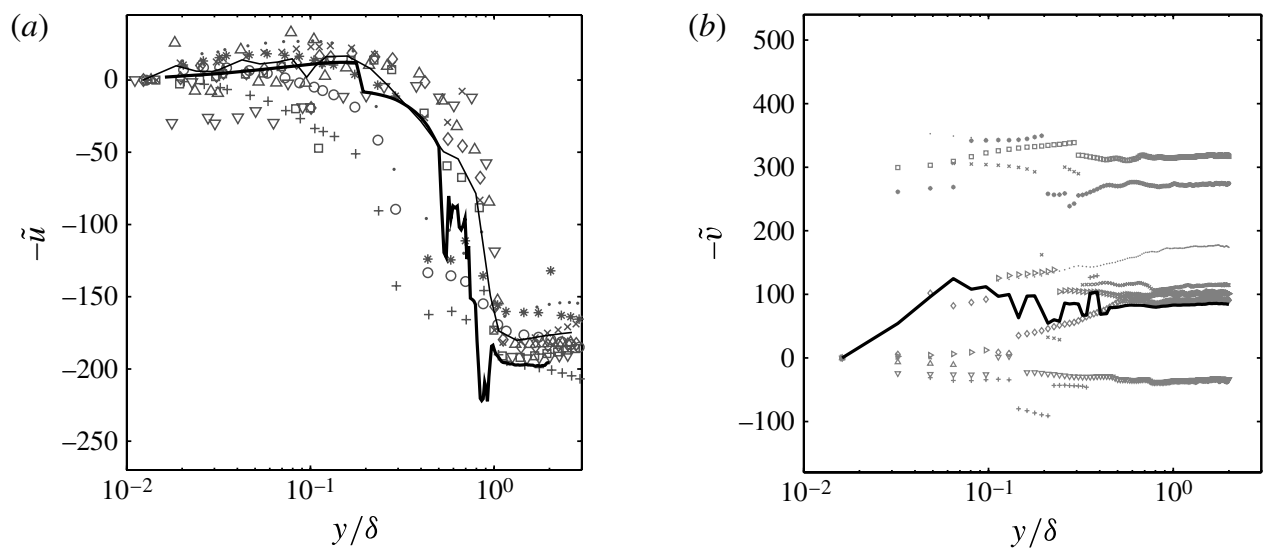

FIGURE 19. (a) The phase variation of $\tilde{u}(y, t)$ in degrees:,$+ x / \delta=0.1 ; \bigcirc, 0.3 ; *, 0.6 ; \cdot, 1.1$; $\times, 2.3 ; \square, 3.4 ; \diamond, 5.0 ; \triangle, 8.4 ; \nabla, 12.1 ;-$, mean for all streamwise locations; bold line is the mean for all PIV runs. $(b) \tilde{v}(y, t)$ : light symbols refer to different PIV runs; bold line is the mean for all PIV.

component of the overall superposition which constitutes the flow which is being energized by the dynamic impulse. It follows then that the experimental mode should be amenable to the same critical layer analogy employed by McKeon \& Sharma (2010).

\section{The forced Orr-Sommerfeld problem}

The linear stability of parallel flows under small perturbations is modelled by the Orr-Sommerfeld equation. The traditional Orr-Sommerfeld problem involves a linearization of the Navier-Stokes equations prior to formulation of the eigenvalue problem. Previously, Reynolds \& Hussain (1972) considered how the Orr-Sommerfeld equation could be adapted to the problem of a turbulent shear flow, where (unlike the laminar flows in transition studies) the nonlinear terms should be significant, but a new 

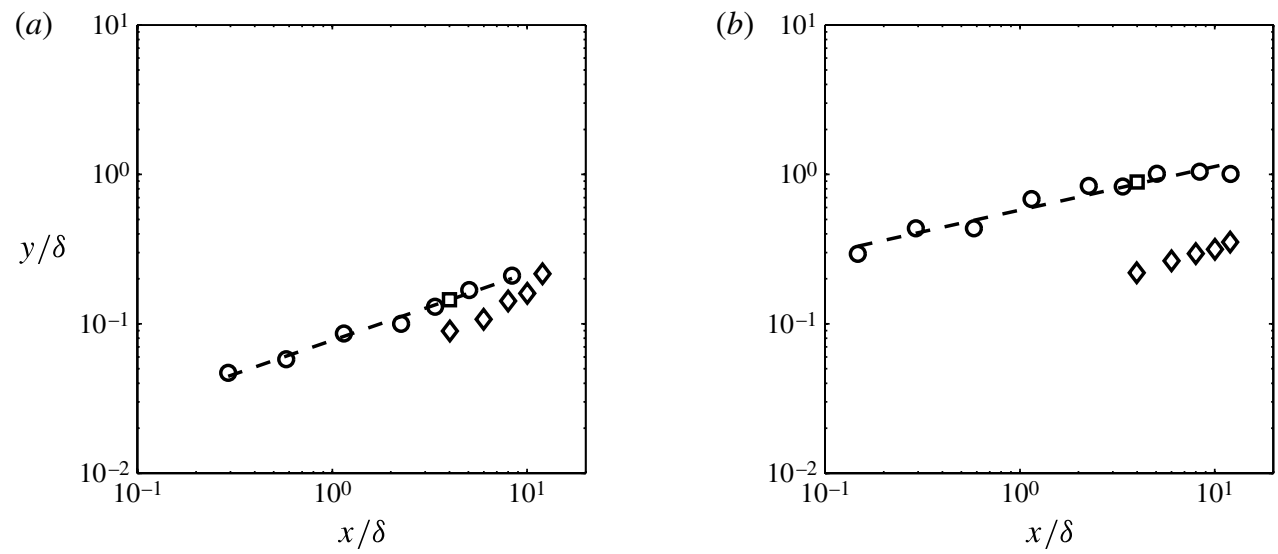

FIGURE 20. (a) The variation in the wall-normal location of the peak amplitude of $\tilde{u}(y, t)$, with range of $0.04<y / \delta<0.21(\bigcirc)$; least-square best fit $(y / \delta)=0.08(x / \delta)^{0.45}(--)$; from PIV ( $\square)$; (b) The variation in the wall-normal location of the $180^{\circ}$ phase shift in $\tilde{u}(y, t)$ (approximated by $\left.\Delta 120^{\circ}\right), \bigcirc$ and - least-square best fit $(y / \delta)=0.58(x / \delta)^{0.29} ; \square$ from PIV. Results from Hussain \& Reynolds (1970) for channel flow (under $100 \mathrm{~Hz}$ perturbation) $(\diamond)$.

approach based on the resolvent operator is considered here. In the following analysis, the significant features of the traditional Orr-Sommerfeld problem are presented, the case of a forced Orr-Sommerfeld problem is introduced, and the differences between these two problems are considered.

\subsection{Formulation}

The governing Orr-Sommerfeld equation is found from substituting a perturbed velocity field,

$$
u(y, t)=U(y)+\tilde{u}(y, t), \quad v(y, t)=\tilde{v}(y, t)
$$

into the Navier-Stokes momentum equation, linearizing the results, and then allowing the perturbation to assume the form of an exponential stream function,

$$
\psi(x, y, t)=\phi(y) \mathrm{e}^{\mathrm{i}(k x-\omega t)},
$$

where the amplitude of the disturbance is purely a function of the wall-normal location. Variables $y, U$, and $k$ are all non-dimensionalized in outer units by $\delta$ and $U_{\infty}$. As defined above, the complex wave velocity is $c=\omega / k=c_{r}+\mathrm{i} c_{i}$. After substituting the perturbation, a fourth-order equation for the amplitude of the propagating disturbance $\phi(y)$ is obtained:

$$
\begin{gathered}
(U(y)-c)\left(\mathrm{D}^{2} \phi(y)-k^{2} \phi(y)\right)-\mathrm{D}^{2} U(y) \phi(y) \\
=\frac{1}{i k R e}\left(\mathrm{D}^{4} \phi(y)-2 k^{2} \mathrm{D}^{2} \phi(y)+k^{4} \phi(y)\right)
\end{gathered}
$$

where $\mathrm{D}=\partial / \partial y$, with physical boundary conditions for the perturbations of no-slip at the wall and decay in the free stream,

$$
\tilde{u}(y, t)=\tilde{v}(y, t)=0, \quad y \rightarrow 0, \infty,
$$

along with parallel flow at the edge of the boundary layer itself. 
Importantly, in the limit of high Reynolds number (the inviscid limit) the governing equation presents a singularity at the point $U\left(y_{c}\right)=c$ where $y_{c}$ is known as the critical point. At the point of the singularity, the amplitude of the disturbance would become infinite in a purely inviscid regime, and thus viscosity must be considered not only near the wall but also in a small domain about the critical point itself, known as the critical layer. It is precisely the critical-layer solution to the Orr-Sommerfeld equation which is of interest in the subsequent analysis, since this critical point occurs under the present forcing, as shown in figure 17. In the following, references to the features of the critical layer indicate those features of the classical, linear, inviscid analysis with a viscous critical layer, as noted in Maslowe (1986), although the method of analysis in the current study does not formally exclude nonlinear behaviour in the way the classical linearization does, due to its use of the resolvent as noted above.

The Orr-Sommerfeld operator $\mathscr{S}$ can be written as a general eigenvalue problem in two parts, following the notation of Reddy, Schmid \& Henningson (1993), with $\mathscr{S}=\mathscr{B}^{-1} \mathscr{A}$ where $\mathscr{A}=(i k R e)^{-1}\left(\mathrm{D}^{2}-k^{2}\right)^{2}-U\left(\mathrm{D}^{2}-k^{2}\right)+\mathrm{D}^{2} U$ and $\mathscr{B}=-\left(\mathrm{D}^{2}-k^{2}\right)$, with the complex eigenvalue $c$, the wavespeed of the disturbance

$$
\mathscr{A} \phi(y)=c \mathscr{B} \phi(y) \text {. }
$$

McKeon \& Sharma (2010) approached the Orr-Sommerfeld operator described above in a similar way (with the use of a three-dimensional divergenceless basis in place of the two-dimensional stream-function approach) but retained the nonlinear terms from the Reynolds decomposition of the velocity field and grouped them into an 'internal' forcing term $f$, as described in $\S 1$. The forced problem then appears as

$$
(c I-\mathscr{S}) \phi(y)=f .
$$

When $f=0$, the linearized (traditional) Orr-Sommerfeld problem is recovered. $f$ can also be generalized to include not only the nonlinear 'internal' forcing, but also external forcing applied to the system. The operator which acts on $f$ is defined as the resolvent (or propagator) $\mathscr{R}$, where

$$
\mathscr{R}=(\sigma I-\mathscr{S})^{-1} .
$$

In this notation, the resolvent set of wavespeeds, $\sigma$, is complementary to the set of eigenvalues $c$ of $\mathscr{S}$, following Kato (1966), since singular values of the resolvent are identically the eigenvalues of the Orr-Sommerfeld operator. When considering the 'internal' forcing by nonlinearities, McKeon \& Sharma (2010) expanded the resolvent operator using a Schmidt decomposition (the continuous form of the singular value decomposition), and wrote the resolvent as

$$
\mathscr{R}=\sum_{n=1}^{\infty} \psi_{n}(y) \rho_{n} \zeta_{n}(y)^{*},
$$

where $\rho_{n}$ are the singular values, and $\psi$ and $\zeta$ are the left and right Schmidt bases. McKeon \& Sharma (2010) showed how consideration of the dominant singular mode of the decomposed resolvent can predict key features of wall-turbulence, without explicit treatment of the closure problem. Turning to the problem of a perturbed turbulent wall-bounded flow, the 'black-box' (or unstructured) forcing in the resolvent treatment now represents both the 'internal forcing' of the nonlinearities in the base turbulent flow, along with the 'external forcing' injected into the flow by the perturbation, and it should be expected that the resolvent method should again identify the singular modes which similarly represent the dynamics of the system. 

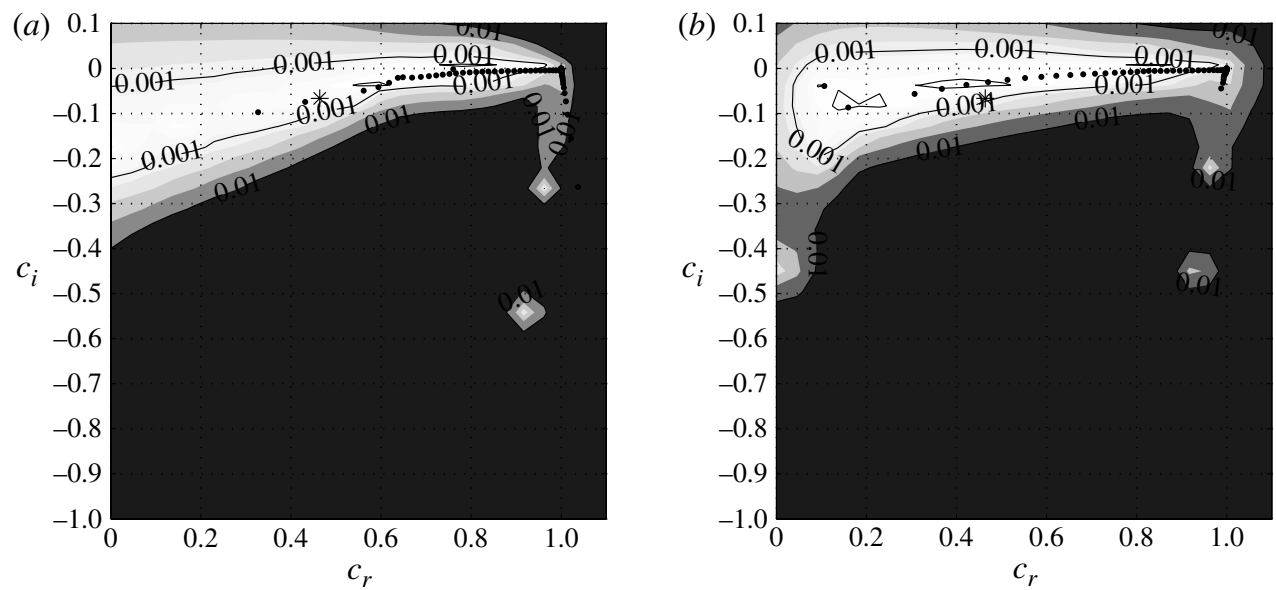

FIGURE 21. Spectrum and pseudospectrum (for $N=100$ ) with contour levels (calculated via the $L_{2}$ norm of the resolvent) at $\epsilon=10^{-5}, 10^{-4}, 10^{-3}$ with decreasing thickness. The eigenvalues are marked as points and the forcing is denoted with an asterisk. The forcing appears near a region of relatively high sensitivity (high-valued norm of the resolvent). The disconnected region is a numerical artifact of the contour routine. (a) Unperturbed velocity profile. (b) Perturbed velocity profile.

\subsection{Mode calculation and comparison}

The Orr-Sommerfeld problem was discretized with a spectral approach utilizing the formulation of the Chebyshev differentiation operators outlined in Weideman \& Reddy (2000). The details of the calculation are provided in the Appendix. The eigenvalue spectrum and pseudospectrum of the resolvent were calculated (figure 21) and indicate that the forcing in the present setup is not only stronger than that used by Reynolds \& Hussain (1972) but also that the forcing is in a region of the eigenspectrum displaying significant sensitivity. Importantly, the region of increased sensitivity due to the nonnormality is quite broad, particularly at higher forcing frequencies, which may allow for a significant range of non-resonant forcing that can still be described adequately by the linear eigenfunctions.

A significant subtlety must also be considered in the analysis of the modified Orr-Sommerfeld operator: the choice of the turbulent velocity profile. The flow under consideration here is neither parallel nor under equilibrium conditions: the importance of these two violations of the fundamental assumptions underlying the analysis will be investigated in what follows. Using the incoming, unperturbed turbulent velocity profile (as done in figure 17) means that the governing operator remains ignorant of the particular non-equilibrium perturbation used in forcing the flow. In contrast, using a perturbed profile allows the operator to take into account the spatially varying effect of the perturbation on the flow field. Both of these calculations were performed in the remaining analysis.

The mode shapes for the most amplified mode were identified by singular value decomposition of the resolvent $\mathscr{R}$ with the experimental forcing, and amplitude and phase results are shown in figures 22 and 23. In addition, the mode shapes for the closest eigenvalue were calculated for comparison between the approaches. Somewhat surprisingly, these two mode shapes appeared to coincide to a significant degree (and were thus excluded from the plots for readability). This appears to be a consequence of the turbulent velocity profile on the Orr-Sommerfeld operator, wherein 
(a)

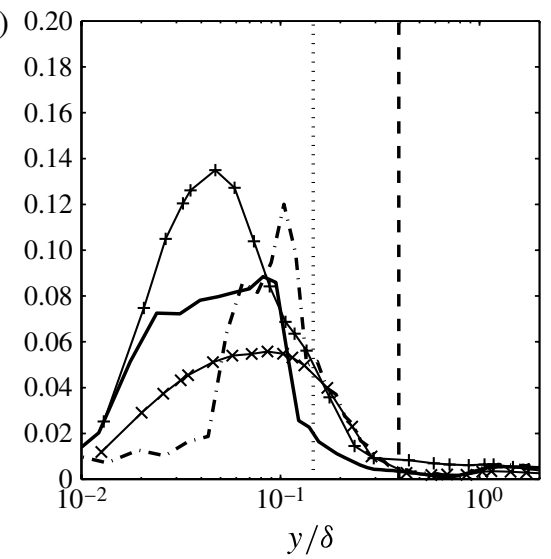

(b)

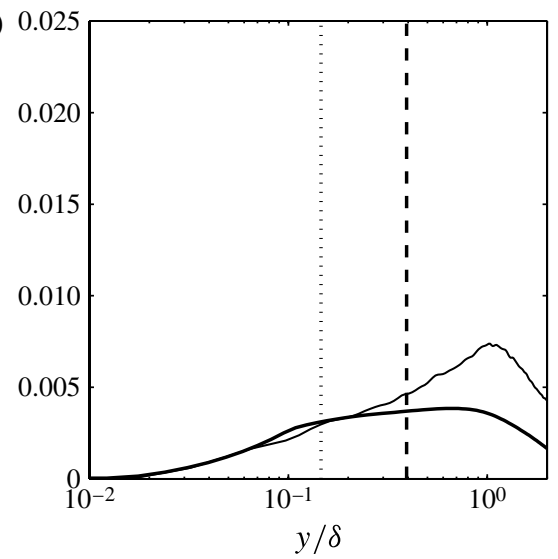

FIgURE 22. (a) The amplitude variation in $\tilde{u}(y, t):--$, from the resolvent analysis using the unperturbed velocity profile; $-\cdots-$, from the resolvent analysis using the perturbed velocity profile; $x / \delta=0.1+; 2.3 \times$ from the experimental hot-wire measurements. $(b)$ The amplitude variation in $\tilde{v}(y, t)$ : - from the resolvent analysis using the unperturbed velocity profile; from the experimental PIV measurement with PIV window centred at $x / \delta \approx 4$. The location of the internal layers, identified in figure 8 , have been marked for the streamwise component: — the first internal layer; $\cdots$ the second internal layer.

(a)

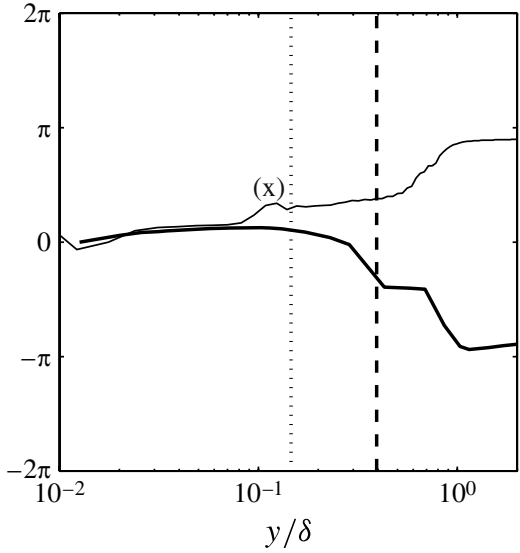

(b)

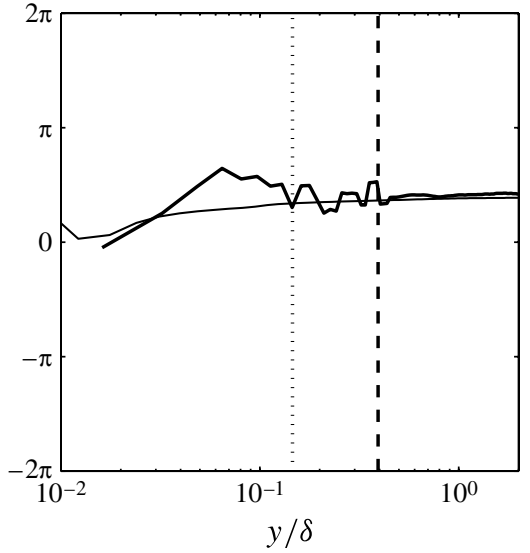

FIgURE 23. (a) The phase variation of $\tilde{u}(y, t)$, with line styles as indicated in figure 22. (b) The phase variation in $\tilde{v}(y, t)$ with markings as above. The ' $\mathrm{x}$ ' marks a distinctive variation in phase which is a robust feature of all Orr-Sommerfeld type solutions.

the distribution of eigenvalues appears to shift closer to the real axis than in the laminar case, thereby lessening the extent of 'non-self-adjointness' of the operator. In the limit of a self-adjoint operator, the singular modes and eigenmodes would overlap, so the fact that the discrete portion of the eigenspectrum of $\mathscr{S}$ tends closer to being real indicates a larger region over which singular and eigenmodes are expected to appear similar.

In order to assess the quality of the predictions by the resolvent method, certain key features of the experimental measures are considered: $(a)$ the peak of the streamwise mode amplitude, which represents the location of the critical layer; $(b)$ the wall- 

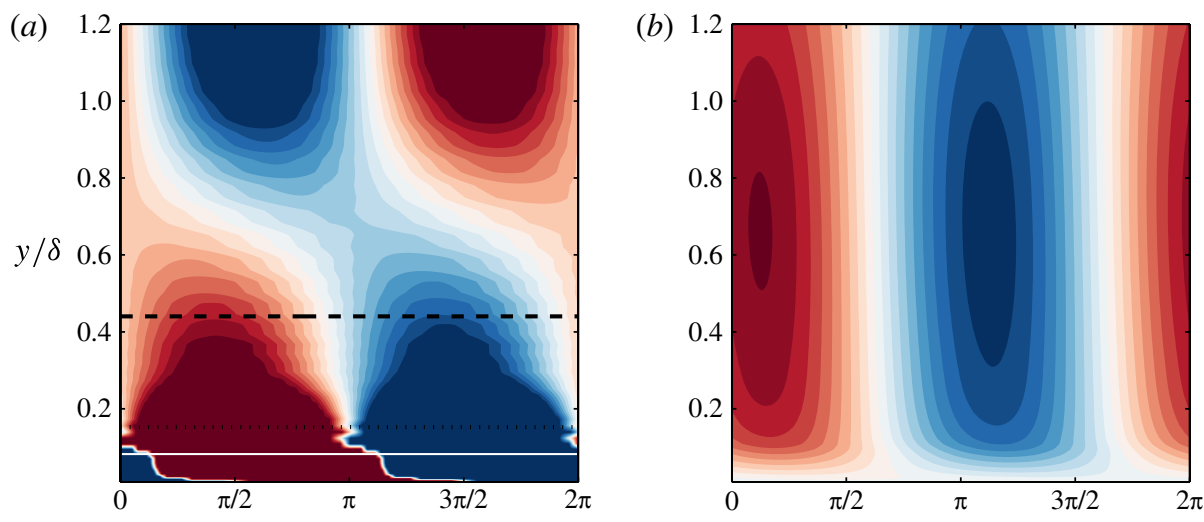

Experimental
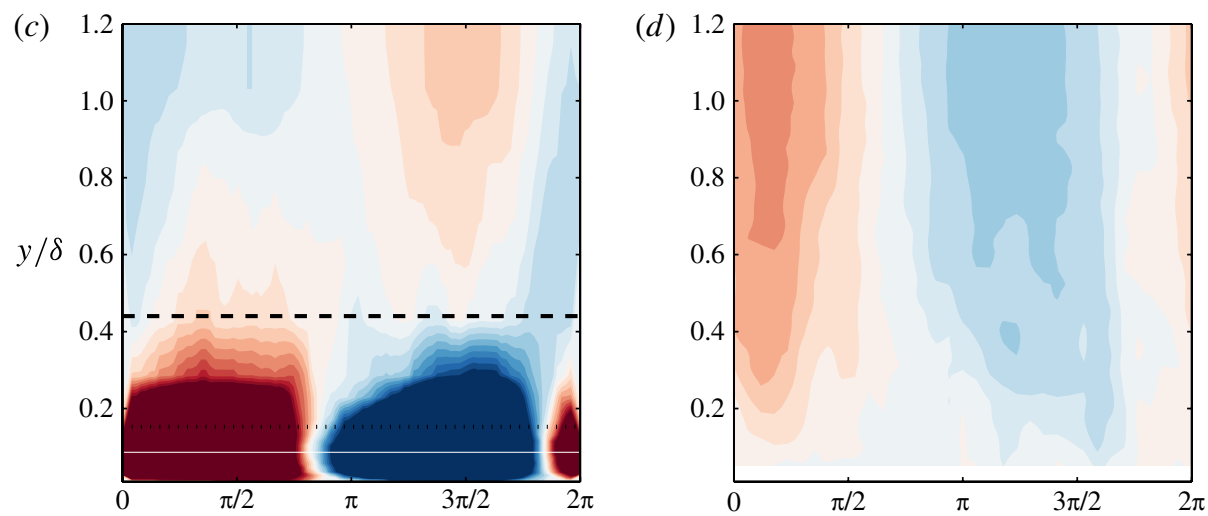

FIgURE 24. Top: (a) A map of the calculated most-amplified singular mode $\tilde{u}(y, t)$ due to the experimental forcing, over an average period $(t \in[0,2 \pi])$. (b) The corresponding map for $\tilde{v}(y, t)$. Bottom: $(c, d)$ The corresponding experimentally measured maps, at $x / \delta \approx 2.3$ assembled via phase locking, as in figure 15.

normal location of the $180^{\circ}$ phase shift in the streamwise mode phase; $(c)$ the relative amplitudes of both mode shapes across the boundary layer; $(d)$ the sense of the phase change across the boundary layer.

(a) The calculated streamwise amplitude variation is shown in figure 22 for resolvent operators employing both the perturbed and unperturbed velocity profiles. The amplitude for the unperturbed profile shows an absolute peak coincident with the experiment at $x / \delta \approx 2.3$, while the overall shape better matches the experiment at the very first streamwise position $x / \delta \approx 0.1$, indicating a critical layer location similar to that predicted in figure 17. This discrepancy is a consequence of the violation of the non-parallel flow assumption, as the boundary layer grows downstream, as well as the non-equilibrium distortion of the mean velocity profile. By employing the perturbed velocity profile in the resolvent operator, this discrepancy can be ameliorated at least partly, in which case the calculated mode shape appears better matched to the experimental shape at $x / \delta \approx 2.3$. However, the difficulty in properly extending the experimental velocity profile to the wall in the perturbed case (as noted in the Appendix) renders the results significantly noisier and, overall, less easily interpreted. 
Using either profile, the magnitude of the peak is significantly overestimated. The sharpness of the predicted peak at the critical layer was a key problem identified by Reynolds \& Hussain (1972) as a failure of the quasi-laminar approach and a justification for explicit treatment of Reynolds stresses. However, even with explicit treatment of the Reynolds stresses, an overestimate of the sharpness of the critical layer peak might be expected on dissipative grounds, due to the local shear within the critical layer. Since viscous dissipation in the turbulent kinetic energy budget scales with shear (including $\partial u^{\prime} / \partial y$, which would not be included in the Reynolds stress term counted in the energy budget of the mean flow), the larger the amplitude of a streamwise disturbance, the higher the shear between the peak of the disturbance and the neighbouring disturbed flow, and the greater the dissipation rate within the disturbance itself. In other words, the region of peak amplitude disturbances is expected to decay faster than regions of lower amplitude, even when Reynolds stresses are accounted for, and in fact Reynolds \& Hussain (1972) found that even including an eddy model closure scheme for the Reynolds stress, the peaks flattened but still failed to match measurements. With this preface in mind, it is clear that the mode shape identified by the resolvent analysis significantly overestimates the sharpness of the critical layer peak, even though the overall shape was captured adequately.

(b) The location of the $180^{\circ}$ phase shift, shown in figure 23 , is captured well in its expected location in the outer region of the boundary layer, as noted in the Appendix. However, it is immediately apparent that the sense of the phase shift is reversed in the experiment from that expected in traditional Orr-Sommerfeld modes. Indeed, there appears to be a close alignment of the phase for wall-normal locations below the location of the second internal layer. In the region between the two internal layers, the phase agreement begins to deteriorate, and beyond the mean edge of the first internal layer the phase trends appears to diverge and become mirror images of one another. That this divergence occurs beyond the second internal layer and that it does not appear to affect the wall-normal location of the phase-shift are both suggestive of the idea that the discrepancy in phase can be explained by the effect of the stress bore, since the location of the $180^{\circ}$ phase shift is a consequence of the crossing of the two inviscid solutions in the asymptotic analysis of the Orr-Sommerfeld problem (noted in the Appendix), and thus is a feature of the outer region of the boundary layer.

(c) The wall-normal amplitude shows reasonable agreement in the region below the first internal layer, and as with the streamwise mode phase, that agreement deteriorates in the region between the two internal layers and shows significant disagreement farther away, where the experimental amplitude is higher. This deviation is expected, since the streamwise turbulent fluctuations of the base flow are significantly stronger than the wall-normal fluctuations, generating greater mixing, and hence the experimental wall-normal amplitude will be suppressed less than the streamwise amplitude. Fitting the predictions to the streamwise amplitude will then necessarily result in a seeming underestimate of the wall-normal amplitude.

(d) As noted above, beyond the first internal layer, the sense of the phase appears to be reversed between the experiments and calculations. However, within the second internal layer, the downstream orientation of the modes is captured quite well. In addition, a distinct feature of the calculated modes, noted by an ' $x$ ' in figure 23 , is a slight reversal in phase just beyond the location of the critical layer. This reversed phase is also seen, although grossly distorted, in the experimental measurements. In the outer region of the boundary layer, where the experimental signals are weaker and the influence of the intermittent edge is more pronounced, the experimental mode shapes indicate an upstream inclination, whereas the predictions show a 
downstream inclination. Considering all three regions of the boundary layer together, the experimental observations suggest crescent-shaped modes, but the predictions indicate sigmoidal shapes, as shown in figure 24. The precise explanation for the phase discrepancy in the streamwise mode outside of the second internal layer is a topic of ongoing investigation. In the wall-normal direction, the phase predictions appear satisfactory, including the inclination at very low wall-normal locations, ignoring some experimental noise.

The resolvent approach does not appear to adequately avoid the incorrect peak amplitude prediction which also afflicted the quasi-laminar eigenmode analysis, although the qualitative prediction of the shape and amplitude was quite accurate and may be sufficient for a variety of potential control applications, which can leverage the simplicity of the resolvent calculation. Where the resolvent calculations performed best was within the second internal layer, where the appropriate sense of phase, and thereby orientation of structures, was predicted. The region between the two internal layers represents flow affected by both the roughness effects and the periodic forcing. Within the second internal layer, however, the roughness effect is largely excluded, but the dynamic forcing persists quite strongly, as shown in figure 14. Therefore, in some sense, the region within the second internal layer represents a region of flow perturbed only temporally, and the resolvent analysis performs reasonably well. This perspective also contributes to the explanation of why using the unperturbed profile achieves better results (besides the difficulty of extrapolating an accurate profile at the wall) - the region in which the results are best is, in fact, largely unperturbed, since it is situated beneath the mean region of the stress bore.

The analysis is significantly complicated by the interplay between the nonequilibrium conditions and the effect of the perturbation itself, aside from the standard challenges of the turbulent boundary layer. However, it is also worth reiterating that the added experimental complication of employing a finite patch of roughness as the forcing mechanism (instead of a thin ribbon) achieves two positive ends: it provides better insight into how a practical forcing mechanism would ultimately behave, and it allows contrast with some of the previous work using wire ribbons, where the weaker forcing was not intimately connected to the mechanism.

The observed spreading of the fluctuating velocity modes and their movement away from the wall - all in violation of the parallel flow assumption - seem to correspond to the observed movement of the stress bore for general non-equilibrium flows, even in the statically perturbed case. These parallels are worthy of future investigation, as they may shed light on the difference between the effects of the re-equilibration process and the changes generated by the dynamic perturbation itself.

Lastly, note that the first singular value output by the resolvent analysis has a magnitude $\rho_{1}=2-5 \times 10^{3}$ (where the exact value depends on the assumed mean profile). Since the resolvent formulation of (5.6) and (5.7) can be thought of as essentially an input-output relationship and the output (the observed velocity mode) has finite amplitude, this suggests that only a tiny component (in the most amplified direction) of the relatively large forcing due to the dynamic roughness input is responsible for the coherent response of the flow. Therefore it could be expected that a smaller wall forcing with optimized coupling to the flow could achieve the same effect, a topic of current investigation. 


\section{Discussion and conclusions}

A dynamic impulse of roughness was used to explore the connection between the fundamental features of impulsive perturbations to turbulent boundary layers, as identified in previous work, and the distinctive effects of introducing an organized wave into the flow field. Experiments provided comprehensive measurements of the flow field, which could be decomposed via phase-locking to illuminate the periodic component. Simplistic analysis based on the forced Orr-Sommerfeld equation using a resolvent approach captured key features of the periodic response of the flow to the forcing, despite the complexity of the flow's return to equilibrium.

In a mean sense, the dynamic impulse manifested a number of the features of the static impulse, from similar internal layers, to deformations of the streamwise statistics, and a partial suppression of the near-wall cycle as observed by spectral composite maps; nevertheless, significant differences warrant special attention. The wave behaviour appeared embedded in the broad shape of the hump in the streamwise turbulence intensity, which was shown to be decomposable into a small-wavelength contribution associated with the roughness impulse and a large-wavelength contribution associated with the organized wave itself. And this distinction between the two contributions of the dynamic impulse was also apparent in the contrast between the integral scale diminution and the spectral composite maps, where turbulence scales were reduced by the roughness impulse, but compensated for by the injection of large scales in the form of the organized wave. These individual observations together suggest that the dynamic impulse provides a much more nuanced and sophisticated method of modifying the turbulent boundary layer, targeting particular structural sizes and specific spectral features of the near-wall cycle. But the most remarkable feature of the dynamic perturbation is the persistence of the wave organization, as shown through the phase-locked decompositions. Not only the mean flow, but also high-order statistics, and the shear stress embodied the key features of the organized wave, as far downstream as was measurable (more than 20 $\delta$ ). From a short impulse, a dynamic stress wave was introduced into the turbulent boundary layer and continued to alter the flow field locally, completely independent of the restored unperturbed boundary condition. This locality of the disturbance provides a powerful insight into the receptivity of the turbulent boundary layer to small modifications.

The organized wave which was forced externally in the flow was observed to excite a critical-layer-type velocity mode in the downstream flow field, despite the fact that the forcing was not in resonance with an eigenmode of the traditional Orr-Sommerfeld equation. This was possible due to the non-normality of the Orr-Sommerfeld operator, which was then subsequently exploited to attempt to predict the mode shapes of the fluctuating velocity components. In contrast to previous studies, which utilized a traditional eigenmode analysis of the quasi-laminar Orr-Sommerfeld operator, the present study utilized a resolvent analysis for the first time in a turbulent boundary layer to describe the velocity fluctuations in terms of the singular modes of the resolvent. Additionally, the input forcing, which in previous work was not significantly stronger than the 'internal forcing' of the nonlinearities of turbulence, was now strong enough that the most amplified singular mode of the resolvent of the Orr-Sommerfeld operator accurately captured significant features of the downstream velocity fluctuations, allowing for some discrepancy due to non-parallel flow effects. The final mode calculations provided reasonably accurate qualitative and quantitative predictions of the velocity fluctuations observed in the perturbed flow, although the over-prediction of the peak sharpness of the critical layer which plagued the quasilaminar studies remained a problem even under the resolvent analysis. In addition, 
disagreement was noted regarding the inclination of the mode shapes in the outer region of the boundary layer.

A number of difficulties confront analytical analysis of the boundary layer perturbation. The non-parallel condition of the flow and the developing nature of the mean turbulent velocity profile both introduce streamwise variation into the analysis which complicates matters significantly. In addition, the non-equilibrium nature of the flow is often difficult to distinguish from what might be considered local but permanent changes to the flow structure. All of these difficulties are likely to contribute to those areas, described in detail above, where the predictions from the Orr-Sommerfeld resolvent analysis disagree with the observations. However, it is important to reiterate that the analysis offered should be viewed as being in the service of interpreting the experimental results, by highlighting those physical features observed which are not easily predicted, and thereby motivating particular areas for further exploration. And thus despite the analytical challenges, the experimental results reported here provide a fresh basis for investigating these questions in more detail. Study of additional frequencies of actuation would provide additional insight into the physics of the flow, although the frequency selected for the present study had the advantage of being largely separable from both the effects of blockage due to an equivalent static roughness and activity associated with the unperturbed boundary layer.

Ultimately, consideration of a dynamic roughness forcing provided a range of new perspectives on how mechanically actuated perturbation of a boundary layer manifests itself in a variety of different flow behaviours: from the generation of a stress bore and its relation to the features of static non-equilibria, to the redistribution of spectral energy intensity. And the significant coherence of the roughness perturbation allowed a phase-locked analysis of the downstream velocity fluctuations, which were then predicted to reasonable accuracy by employing a resolvent approach which, despite suffering some of the same deficiencies as earlier quasi-laminar methods, is widely understood to be better suited to high-Reynolds-number turbulent flows.

This work is supported by the Air Force Office of Scientific Research Hypersonics and Turbulence portfolio, under grant no. FA9550-08-1-0049 (Program Manager J. Schmisseur). Also, the authors wish to thank C. Gonzalez of California Polytechnic University Pomona for assistance with the PIV setup, as well as M. Guala of the Graduate Aerospace Laboratories at the California Institute of Technology for assistance in preparing the wind tunnel for the current experiments. In addition, the authors thank the reviewers for their very helpful comments and corrections, particularly regarding the spectral calculations, which significantly improved this manuscript.

\section{Appendix}

The non-normality of the Orr-Sommerfeld operator, described in the introduction $\S 1$, is manifested in the sensitivity of its eigenvalues to small perturbations, and the degree of that sensitivity provides a means of measuring the level of non-normality. Reddy et al. (1993) outlined the most intuitive method for measuring this degree of non-normality through the calculation of a 'pseudospectrum' defined by the levelcurves of the norm of the resolvent. The pseudospectrum indicates the extent of the region of high-sensitivity about each eigenvalue and makes clear that simply 
calculating the eigenvalues in the traditional way may not be meaningful without also calculating the accompanying pseudospectrum.

To solve the eigenvalue problem, a spectral approach using Chebyshev collocation matrices was employed. The calculation was first performed on the Blasius solution of the laminar boundary layer and the least-damped eigenvalues at a variety of Reynolds numbers were calculated and compared to those found by Jordinson (1970) and Danabasoglu \& Biringen (1989). The collocation matrix dimension is $N$. The eigenvalues were matched to five decimal places with $N \geqslant 40$. Besides this leastdamped eigenvalue, the resolution of other eigenvalues is more difficult. In particular, the presence of non-physical (spurious) eigenvalues under Chebyshev methods is a well-known consequence of the discretization and has been discussed in Boyd (2000). To eliminate the spurious values, the approach of Stewart et al. (2009) was adopted, in which the adjoint problem is simultaneously solved and the corresponding complex-conjugate eigenvalues are compared to those of the original problem; then non-overlapping values are eliminated as spurious.

Extending this approach from the Blasius laminar boundary layer to the experimental turbulent boundary layer presents another challenge regarding handling the velocity field closer to the wall than could be resolved by the hot-wire measurement. The approach of Spalding (1961) was employed to extend the experimental turbulent boundary layer profile to the wall. However, Spalding's method relies on the measurement of the friction velocity $u_{\tau}$, which is difficult to ascertain for the impulsively perturbed case. It was shown above that the friction velocity tends to decrease immediately downstream of the perturbation and then recover slowly, but the magnitude of that decrease was not measurable with confidence. Therefore, the choice of $u_{\tau}$ for the Spalding extension was iterated until the velocity profile appeared continuous $\left(u_{\tau}=2 / 3 u_{\tau, 0}\right.$ with $\kappa=0.41$ and $C=4.9$ following Jacobi \& McKeon 2011).

With the experimental turbulent boundary layer in the standard Orr-Sommerfeld operator, the eigenvalues, eigenmodes, and pseudospectrum were calculated. The complexity of the turbulent boundary layer, due both to the matching with the Spalding fit near the wall and also experimental noise, meant that significantly larger collocation matrices were needed to produce smooth eigenmodes and converged eigenvalues. It was found that $N \gtrsim 100$ assured convergence for the least-damped eigenvalues and produced smooth eigenmode shapes. The danger of overly dense collocation matrices distorting the results near the boundaries was investigated, but trends seemed to smoothly approach convergence in the laminar test case for $N \approx 100$, which has been used successfully in a variety of other studies (e.g. Schmid \& Henningson 2001). Therefore, $N$ was fixed with an upper bound of 120 in the current study to avoid distortion, despite an observed dependence of the shape of the criticallayer peak on $N$. In general, resolving the spectrum for a turbulent wall-bounded flows is quite difficult, as noted by McKeon \& Sharma (2010).

In the pseudospectra (figure 21), the small vertically oriented collection of eigenvalues near $c_{i}=1$ is just a segment of the poorly resolved continuous portion of the spectrum, which Grosch \& Salwen (1978) showed should span the line $c_{i}=1$ for boundary-layer problems. The distribution of other eigenvalues near the real axis is a consistent feature of the calculations - increasing resolution tends to increase the distance to the real axis slightly, but the overall trend is preserved largely independent of $N$, a feature which may help explain the general similarity between singular and eigenmodes identified above. 
Finally, a key concept regarding critical layers is worth reiterating. There are two defining characteristics of the streamwise critical-layer eigenfunction: an amplitude peak near the location of the critical point, $y_{c}$, and a phase shift of $180^{\circ}$ somewhere in the outer region of the boundary layer. A number of classic sources could be easily misinterpreted to suggest that the phase shift should also occur at the critical point itself (Schlichting 1968; Hinze 1975), but this is not correct. Schlichting's asymptotic analysis reveals that the phase shift is a purely inviscid phenomenon, and it occurs where the two inviscid solutions (of the four total solutions) to the Rayleigh equation meet, far from the inner region of the boundary layer. Therefore, these two locations, $y_{c}$ near the critical point and $y_{p}$ at the phase shift, are expected to be distinct and physically significant. The former represents the centre of the critical layer itself, while the latter represents the end of the inviscid solutions, which are not valid too far from the critical point, about which they can be expanded in series.

\section{REFERENCES}

Andreopoulos, J. \& Wood, D. H. 1982 The response of a turbulent boundary layer to a short length of surface roughness. J. Fluid Mech. 118, 143-164.

Antonia, R. A. \& LuXton, R. E. 1971 The response of a turbulent boundary layer to a step change in surface roughness Part 1. Smooth to rough. J. Fluid Mech. 48 (4), 721-761.

Antonia, R. A. \& LuXton, R. E. 1972 The response of a turbulent boundary layer to a step change in surface roughness Part 2. Rough-to-smooth. J. Fluid Mech. 53 (4), 737-757.

Boyd, J. P. 2000 Chebyshev and Fourier Spectral Methods. Dover.

BuiltJes, P. J. H. 1975 Determination of the Eulerian longitudinal integral length scale in a turbulent boundary layer. Appl. Sci. Res. 31.

Danabasoglu, G. \& Biringen, S. 1989 A Chebyshev matrix method for spatial modes of the Orr-Sommerfeld equation. NASA Tech. Rep. CR 4247.

Grosch, C. E. \& SALWEN, H. 1978 The continuous spectrum of the Orr-Sommerfeld equation. Part 1. The spectrum and the eigenfunctions. J. Fluid Mech. 33-54.

Hellström, L. H. O. \& SMits, A. J. 2011 Visualizing the very-large-scale motions in turbulent pipe flow. Phys. Fluids 23; 011703.

HinZE, J. O. 1975 Turbulence, 2nd edn. McGraw-Hill.

Hussain, A. K. M. F. \& ReYnolds, W. C. 1970 The mechanics of an organized wave in turbulent shear flow. J. Fluid Mech. 41, 241-258.

Hussain, A. K. M. F. \& Reynolds, W. C. 1972 The mechanics of an organized wave in turbulent shear flow. Part 2. Experimental results. J. Fluid Mech. 54, 241-261.

Hutchins, N. \& Marusic, I. 2007 Large-scale influences in near-wall turbulence. Phil. Trans. R. Soc. Lond. 365, 647-664.

JACOBI, I. \& MCKEON, B. J. 2011 New perspectives on the impulsive roughness-perturbation of a turbulent boundary layer. J. Fluid Mech 677, 179-203.

Jordinson, R. 1970 The flat plate boundary layer. Part 1. Numerical integration of the Orr-Sommerfeld equation. J. Fluid Mech. 43, 801-811.

KATO, T. 1966 Perturbation Theory for Linear Operators. Springer.

Maslowe, S. A. 1986 Critical layers in shear flows. Annu. Rev. Fluid Mech. 18, 405-432.

McKeon, B. J. \& Sharma, A. S. 2010 A critical layer model for turbulent pipe flow. J. Fluid Mech. 658, 336-382.

Monty, J. P., Hutchins, N., NG, H. C. H., Marusic, I. \& Chong, M. S. 2009 A comparison of turbulent pipe, channel and boundary layer flows. J. Fluid Mech. 632, 431-442.

MORRISON, J. F. 2010 Boundary layers under strong distortion: an experimentalist's view. In Prediction of Turbulent Flows. Cambridge University Press.

Pearson, B. R., Elavarasan, R. \& Antonia, R. A. 1997 Effect of a short roughness strip on a turbulent boundary layer. Appl. Sci. Res. 59 (1), 61-75. 
Reddy, S. C., Schmid, P. J. \& Henningson, D. S. 1993 Pseudospectra of the Orr-Sommerfeld operator. SIAM J. Appl. Maths 53, 15-47.

ReYnolds, W. C. \& Hussain, A. K. M. F. 1972 The mechanics of an organized wave in turbulent shear flow. Part 3. Theoretical models and comparisons with experiments. J. Fluid Mech. 54, 263-288.

SCHLichting, H. 1950 Amplitude distribution and energy balance of small disturbances in plate flow. NACA Tech. Rep. 1265.

Schlichting, H. 1968 Boundary-Layer Theory, 6th edn. McGraw-Hill.

Schmid, P. J. \& Henningson, D. S. 2001 Stability and Transition in Shear Flows. Springer.

SChubAuer, G. B. \& SKRAMSTAD, H. K. 1943 Laminar-boundary-layer oscillations and transition on a flat plate. NACA Tech. Rep. 9595 .

Schubauer, G. B. \& Skramstad, H. K. 1947 Laminar boundary-layer oscillations and stability of laminar flow. J. Aeronaut. Sci. 14 (2), 69-78.

Smits, A. J. \& Wood, D. H. 1985 The response of turbulent boundary layers to sudden perturbations. Annu. Rev. Fluid Mech. 17, 321-358.

Smits, A. J., Young, S. T. B. \& Bradshaw, P. 1979 The effect of short regions of high curvature on turbulent boundary layers. J. Fluid Mech. 94, 209-242.

Spalding, D. B. 1961 A single formula for the law of the wall. J. Appl. Mech. 28, 455.

Stewart, P. S., Waters, S. L., Billingham, J. \& Jensen, O. E. 2009 Spatially localised growth within global instabilities of flexible channel flows. In Seventh IUTAM Symposium on Laminar-Turbulent Transition.

Townsend, A. A. 1961 Equilibrium layers and wall turbulence. J. Fluid Mech. 11, 97-120.

Trefethen, L. N. \& Embree, M. 2005 Spectra and Pseudospectra: The Behavior of Nonnormal Matrices and Operators. Princeton University Press.

Weideman, J. A. C. \& Reddy, S. C. 2000 A MATLAB differentiation matrix suite. ACM Trans. Math. Softw. 26 (4), 465-519. 